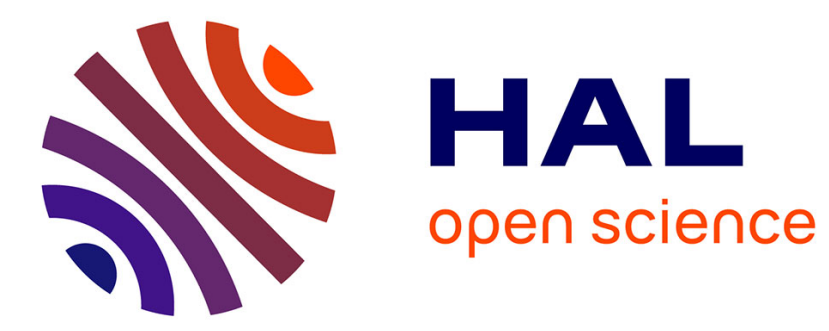

\title{
L'aménagement du territoire et la notion de frontière à l'époque ancienne
}

\author{
Paola Calanca
}

\section{To cite this version:}

Paola Calanca. L'aménagement du territoire et la notion de frontière à l'époque ancienne. Extrême-

Orient Extrême-Occident, 2006, 28 (28), pp.57-93. 10.3406/oroc.2006.1224 . halshs-02512995

\section{HAL Id: halshs-02512995 \\ https://shs.hal.science/halshs-02512995}

Submitted on 20 Mar 2020

HAL is a multi-disciplinary open access archive for the deposit and dissemination of scientific research documents, whether they are published or not. The documents may come from teaching and research institutions in France or abroad, or from public or private research centers.
L'archive ouverte pluridisciplinaire HAL, est destinée au dépôt et à la diffusion de documents scientifiques de niveau recherche, publiés ou non, émanant des établissements d'enseignement et de recherche français ou étrangers, des laboratoires publics ou privés. 


\section{L'aménagement du territoire et la notion de frontière à l'époque} ancienne

Paola Calanca

\section{Citer ce document / Cite this document :}

Calanca Paola. L'aménagement du territoire et la notion de frontière à l'époque ancienne. In: Extrême-Orient, ExtrêmeOccident, 2006, $\mathrm{n}^{\circ} 28$. Desseins de frontières. pp. 57-93.

doi : 10.3406/oroc.2006.1224

http://www.persee.fr/doc/oroc_0754-5010_2006_num_28_28_1224

Document généré le 28/09/2015 


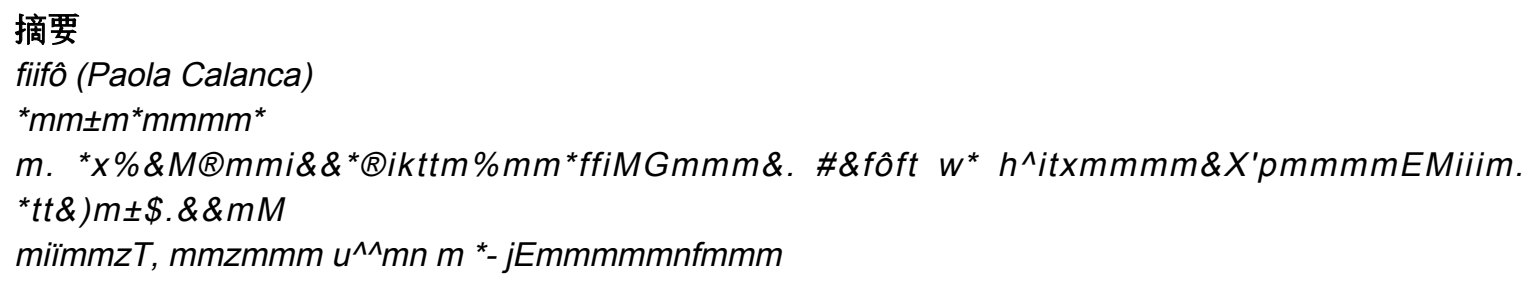

\begin{abstract}
Territorial Planning and the Notion of Border in Ancient Times

The ancient texts show the link between the archaic foundation of the social order and marks, lines, and boundaries drawing and fixing the outline of a social sphere organized and subject to a single authority. In this article, the author emphasizes the importance of the notion of border in Chinese political tradition in ancient times, the terminology of which is already to be found in Shang oracular inscriptions and Western Zhou bronzes. Ancient territorial units were organized around two devices: the Altar of the Earth in the center and the borders at the margins of the territory. The Altar of the Earth of a locality is the emblem of a horizontal relation that links the community with the great square of the nine provinces and of a vertical relation which places it under the authority of the State, illustrated by the Taiyi talisman found in Mawangdui. As for the territory of the unified Empire, its borders were most likely marked by visible signs, even on the southern border which did not require as considerable an organization as the one set up in the North.
\end{abstract}

\title{
Résumé
}

Les textes anciens établissent un lien étroit entre les fondements archaïques de l'ordre social et l'établissement de bornes, de bordures et de limites destinées à marquer et à dessiner les contours de l'espace social organisé et soumis à une même autorité. Cet article s'attache à rappeler l'importance de la notion de frontière dans la tradition politique chinoise à l'époque ancienne, dont la terminologie se retrouve déjà dans les inscriptions oraculaires des Shang et sur les bronzes des Zhou de l'Ouest. Les unités territoriales anciennes sont organisées autour de deux dispositifs : l'autel du sol au centre et les frontières à l'extrémité du territoire. L'autel du sol d'une localité est l'emblème d'une relation horizontale qui relie la communauté au grand carré des neuf provinces et d'un rapport vertical qui la place sous l'autorité de l'État, illustré par le talisman de Taiyi trouvé à Mawangdui. Quant au territoire de l'empire unifié, ses limites étaient vraisemblablement marquées par des signes visibles, même sur sa frontière méridionale qui ne nécessitait pas un dispositif aussi important que celui édifié au Nord. 


\title{
L'aménagement du territoire et la notion de frontière à l'époque ancienne
}

\author{
Paola Calanca*
}

[Dans les temps anciens], le ciel était sec et la terre fendillée. Mâles et femelles n'étaient pas distingués et les animaux vivaient dans la cruauté. Le peuple habitait dans des huttes de branchages et n'avait nulle place qui fût à lui. Ceux qui mouraient de froid ou de faim étaient roulés dans des nattes et reposaient les uns sur les autres [...]. Alors on commença à diviser les montagnes, les rivières, les ravins et les vallées, et à établir des tracés carrés de terres fertiles. Le nombre d'habitants fut calculé et chacun se vit attribuer une place dans l'espace et selon le nombre. On bâtit des murailles et on creusa des fossés, on établit des équipements et des obstacles défensifs [...]'

Apprécier une notion aussi complexe que celle de frontière au sujet d'un pays dont le passé étatique plonge ses racines au toumant du $\mathrm{II}^{\mathrm{e}}$ millénaire avant notre ère mérite un retour sur image, car cette extrême longévité s'accompagne d'une aura civilisatrice aux limites très étendues. L'idée d'une sphère d'influence dépassant les frontières «nationales" et rendant éventuellement celles-ci caduques a eu des répercussions non seulement dans le domaine culturel, mais également sur la vision politique, au point que parfois l'esprit chinois a pu paraître, à certains, rétif à la notion de frontière et les confins de l'empire dépourvus de consistance réelle. Il est vrai, par ailleurs, qu'à la fin du $\mathrm{XIX}^{\mathrm{e}}$ siècle, certains réformistes chinois ont développé des idées universalistes, tel par exemple Tan Sitong (1865-1898):

Le gouvernement global s'étendra sur un seul monde unifié et verra la fin de toute différence entre les États [...] car un homme qui jouit de sa pleine et entière liberté ne saurait accepter de n'être que le citoyen d'un seul État. Les tracés des frontières s'effaceront. Les guerres s'arrêteront. Nul ne nourrira plus de soupçon quant aux intentions de son voisin. Complots et stratégies n'auront plus cours. Nulle différence 
n'existera plus entre mon bien et celui des autres : l'égalité régnera entre tous. Quand le monde sera ainsi gouverné, nul ne prêtera plus attention à son gouvernement. Ce sera la fin des rois, des maîtres et de toute la hiérarchie sociale. L'administration ne sera plus guidée que par le bien commun et ce sera la fin de toutes les différences de fortune. Mille villages, dix mille quartiers ne formeront plus qu'une seule famille. Chacun lors de ses déplacements sera considéré comme un invité honoré dans son pays d'accueil. Chaque homme sera pour tous un compatriote. Les pères n'auront plus besoin de s'efforcer de traiter leurs enfants avec bonté, ni les enfants de servir leurs parents avec piété filiale, ni les frères de se traiter avec amitié, ni l'époux et l'épouse de décider qui commande à la maison. C'est cela qu'appelle de ses voeux un livre occidental paru il y a à peine un siècle et cela aussi qui ressemble beaucoup au passage du chapitre «Mouvement des rites» où il est question du règne de la «grande unité» (datong) ${ }^{2}$.

L'utilisation dans les programmes des réformistes du $\mathrm{XIX}^{\mathrm{e}}$ siècle et des révolutionnaires républicains du siècle dernier d'un passage du chapitre «Mouvement des rites» («Liyun») du Mémoire sur les rites, « au temps où la grande voie était en action ${ }^{3}$, qui rendit célèbre dans les milieux occidentaux la notion universaliste de «grande unité», ne doit toutefois pas tromper. Un monde sans frontières n'est pas un idéal de référence dans les textes chinois anciens. La plupart d'entre eux, au contraire, établissent un lien étroit entre les fondements archaïques de l'ordre social et l'établissement de bornes, de bordures et de limites destinées à marquer et à dessiner les contours de l'espace social organisé et soumis à une même autorité. Les découvertes archéologiques confirment, plus qu'elles n'infirment, une partie des événements qui ont marqué l'antiquité chinoise, telle qu'elle a été transmise par la tradition classique, et autorisent ainsi une première quête au sujet de la notion de frontière avant l'époque impériale.

\section{Préliminaires}

\section{La terre, ou l'ordre carré}

Le développement de l'État chinois, tout comme celui du monde grec, s'est accompagné de l'établissement de réseaux complexes de limites et de bordures définissant l'ordre social et spatial des communautés qui l'habitaient, et dessinant les contours d'un monde différencié. Dans les sources retraçant l'origine de la vie communautaire, la frontière se présente comme le dessin extérieur d'un travail de civilisation, telle qu'elle apparaît dans le passage du Maître de Huainan, cité cidessus en exergue, ou encore plus clairement dans les Mémoires historiques:

Jadis, le peuple habitait dans des gorges profondes et des grottes et ne connaissait pas le travail des champs. [...] Le monde était un chaos [...]. La terreur régnait partout [...]. Puis vinrent les sages qui distinguèrent les différentes espèces et veillèrent à ce qu'elles n'empiètent pas sur leur territoire respectif. Les animaux mâles et femelles furent relégués dans les plaines et les montagnes, les oiseaux des deux genres dans les forêts et les marais, les poissons et autres créatures à écailles trouvèrent leur place dans les 
cours d'eau au fond des vallées. Alors le peuple fut gouverné. On édifia les murailles intérieures et extérieures des cités. Dans leurs enceintes il y eut des ruelles et des îlots. A l'extérieur des cités s'alignèrent en ordre quadrillé les champs et les chemins de mille et de cent qui les bordaient. Hommes et femmes devinrent maris et épouses. Ils se virent attribuer chacun un champ et une maison et celles-ci s'alignèrent en rangées dans les agglomérations. Des cartes et des registres furent établis et le nom de chaque famille fut inscrit sur des rôles. Une hiérarchie des fonctions fut établie et il y eut des fonctionnaires petits et grands à qui l'on décerna des titres et des salaires. Avec le chanvre et la soie on tissa des vêtements et les cinq céréales devinrent la nourriture de chacun et chacun s'affaira à labourer et à sarcler, à bêcher et à désherber. Le palais connut alors les saveurs qui lui étaient agréables, l'œil put se réjouir de la beauté et le corps connaître les avantages du confort. Mais il n'est rien dans tout cela qui n'ait été le fruit de l'effort et du travail humain 4 .

Les bordures territoriales citées dans les textes anciens sont variées et de natures diverses : tracé des limites des cités et de la campagne qui en dépend; tracé des limites du domaine royal et de celui des feudataires censés être ses remparts, mais surtout tracé fondamental entre le peuple des Xia et celui des peuples extérieurs. Le célèbre site de Banpo offre une représentation plus concrète de ces premiers ordonnancements de la société, et surtout des premières séparations entre groupes humains, que ne le rapportent les deux sources plus tardives citées ci-dessus. Sur une surface de $30000 \mathrm{~m}^{2}$, les archéologues ont mis au jour, près de $\mathrm{Xi}$ 'an, un habitat néolithique datant du ve millénaire avant notre ère et comprenant des fondations d'habitations et des vestiges de fours et d'enclos à bétail. Le village est ceint par un fossé de cinq à six mètres de profondeur et de six à huit mètres de largeur visant à assurer la sécurité de la population. L'une des spécificités du site est constituée par un fossé transversal, profond d'un mètre et demi et large de deux mètres, qui scinde le village en deux parties avec, dans chacune d'entre elles, les restes des fondations d'une grande maison, indiquant peut-être la cohabitation dans cette localite de deux groupes distincts d'habitants ${ }^{5}$.

Avant d'analyser plus en détails l'aménagement du territoire et la vision des frontières qu'il implique, il est indispensable de s'attarder sur le cadre politique dans lequel il s'inscrit, et de rappeler brièvement l'organisation étatique qui le sous-tend et les deux entités autour desquelles se régulent la société et l'espace, temporel et spatial, chinois : le cercle (le ciel) et le carré (la terre). Ces deux entités fixent le cadre dans lequel il convient aussi de situer l'idéologie de l'aménagement du territoire, qui ressemble fort à une transposition spatiale de l'ordre politique :

Le ciel est animé d'un mouvement en cercle comme les astres et les planètes qui tournent autour de l'étoile polaire. [...] Le prince dans son gouvernement prend modèle sur le ciel et fait en sorte que les planètes et les étoiles [ses ministres et ses conseillers] ne tournent qu'autour d'un seul pôle. 
Le commentaire de Kong Yingda (574-648) ajoute:

La terre distingue cinq espèces de sols d'où naissent toutes sortes de productions différentes. Le prince prend modèle sur la terre et nourrit toutes sortes de talents differents ${ }^{6}$.

En dépit d'un calcul simplifié des relations entre cercle et carré (la proportion 3/4), la figure du cercle et, par conséquent, le ciel qui lui répond gardent leur mystère.

Le ciel est un cercle de $360^{\circ}$, réparti en douze stations de $30^{\circ}$ chacune. Tel est son nombre entier. Mais il reste encore cinq jours et quart pour faire une année et ceci est son impair. Si les degrés célestes ne comptaient pas d'impair, même un enfant pourrait calculer le calendrier mille ans à l'avance sans la moindre difficulté. Mais à cause de cet impair, de ce reste qui dépasse les nombres entiers, le calcul du calendrier garde son mystère?.

Ce passage de la grande encyclopédie Mer de jade (Yuhai), compilée par Wang Yinglin (1223-1296), met en lumière l'importance du cycle calendérique, nécessaire pour assurer la correspondance entre l'année civile et les diverses tâches agricoles. Les difficultés rencontrées émanent de l'instabilité des calculs de périodicité des conjonctions entre la rotation de la terre et les cycles des corps célestes, déjà soulignées dans un chapitre du Guanzi :

L'ordre des mouvements célestes du yin et du yang ne tombe pas juste, les différences de ses restes et de ses manques ne peuvent pas être complétées [et sont incluses dans le calcul de son mouvement]. [...] Ce qui permet d'établir une administration ordonnée, c'est la terre. La terre dont les tracés et les produits doivent être nettement délimités ${ }^{8}$.

Si les calculs du calendrier sont le domaine des restes et des fractions et doivent être périodiquement repris faute d'arriver à un nombre entier satisfaisant, la terre (di), pareille à un échiquier, est le domaine des tracés visibles, des bornes, des levées de terre, des fossés, des rideaux d'arbres, des murs et des portes des îlots d'habitation ou des murailles des cités dans lesquelles la population a élu sa demeure. Tout comme l'échiquier, elle est composée de cases carrées. Sa définition dans l'un des premiers dictionnaires - le Shuowen jiezi - en fait le support du déploiement [et du classement] dans l'espace (chen lie) des dix mille êtres (wan wu $)^{9}$, analogue en cela au terme grec «cosmos» qui désigne, tout comme le terme chen lie, l'ordre des armées. Le carré de la terre est donc divisible à l'infini et permet d'attribuer, à l'intérieur de ses subdivisions, un territoire à chaque espece qui l'habite, évitant ainsi les empiètements mutuels ${ }^{10}$, comme le soulignent les préceptes d'un théoricien du bon gouvernement, Xunzi (ca. $-298 /-235)$ :

L'homme ne peut se passer de vivre en société. Une société où les parts [de chacun] ne seraient pas définies engendrerait des luttes, les luttes le désordre, le désordre la misère. 
Ainsi, ne pas avoir sa part est pour l'homme un grand malheur, tandis que c'est dans la distribution que réside le grand profit de l'empire, et le prince veille à être le seul à détenir le pouvoir de distribution".

\section{L'architecture de l'État}

L'ordre politique sur lequel repose le régime d'abord royal, puis impérial chinois, donne au nombre impair une fonction centrale. L'impair est l'attribut du Ciel $^{12}$ et du roi :

Le fils du Ciel n'a pas d'épouse [qui soit son égale], aussi le déclare-t-on sans pair. Le fils du Ciel n'est jamais reçu dans l'espace entre les quatre mers selon les rites de l'invité [il est partout l'hôte], aussi le déclare-t-on sans maître ${ }^{13}$.

Cette conception du «un» trouve son application en miniature dans le groupe de «cinq $(w u)$, module d'organisation de la population. Quatre hommes et un chef pour les commander, cinq familles, dont celle de leur chef, solidairement responsables : ce système était sans doute en usage depuis la haute antiquité parmi les habitants des zones urbaines et périurbaines, décimalisés pour les levées militaires. Bien que certainement idéalisé dans ses finalités et son bon fonctionnement, il fut progressivement généralisé à l'ensemble des territoires à l'époque des Royaumes Combattants (-453/-222) et devint la cellule de base de l'empire unifié des Qin (-221/-206) et des Han (-206/220).

Dans les champs et les îlots ruraux, la population est organisée par groupes de cinq et ce sont des unités semblables qui, dans les armées, sont inscrites sur les rôles et reçoivent les ordres dont ils sont solidairement responsables ${ }^{14}$.

Le nombre «cinq», considéré comme optimal dans cette ergonomie primitive, sert aussi à contrôler les quatre directions de l'espace à partir d'un centre, qu'il $s$ 'agisse de la disposition spatiale des armées ${ }^{15}$ ou de l'organisation des groupes d'habitations, comme on le lit explicitement dans les plaquettes en bambou d'époque Qin (-221/-206) de Shuihudi :

Si un voleur pénètre dans la maison de $X$, lui inflige une blessure et que $X$ crie : «Au voleur", et que ses quatre voisins, le chef de son îlot et le chef de son groupe de cinq ne sont pas chez eux et n'entendent pas les cris, faut-il les poursuivre? Si les quatre voisins n'étaient pas là, non ; mais, pour le chef du groupe de cinq et le chef de l'îlot, il faut intenter des poursuites à leur encontre s'ils étaient absents (fiche 98);

Qu'appelle-t-on ses «quatre voisins" en termes juridiques? Ce sont les familles appartenant à un même groupe de cinq (fiche 99) ${ }^{16}$.

Il est loisible d'identifier la fonction du «un» au roi, «l'homme un» (yiren) appellation déjà citée dans les inscriptions oraculaires des Shang ( $\mathrm{XVI}^{\circ} \mathrm{s}$.-milieu du $\mathrm{Xr}^{\mathrm{e}}$ avant notre ère) -, et à sa fonction de garant du territoire qui lui est soumis. Quatre directions, mais aussi quatre limites et un centre pour les contrôler, concept 
que l'on trouve concrétisé dans le sacrifice à l'autel du sol, le centre emblématique d'un territoire. La pratique d'ériger des «places centrales» lors de la fondation des cités est attestée depuis les Shang, telle celle établie dans la région de Lu à l'époque de Wuding (ca. début XIII $s$. avant notre ère) d'après une inscription divinatoire:

Question: le roi se rend à Lu. (He 8221.)

Question: le roi se rend à Lu et y établit un poste militaire. (He 8219.)

Divination du jour jihai, devin Nei : le roi fait dresser une pierre au nord-est de Lu et là il établit une cité. (He 13505, face ${ }^{17}$.).

Selon la tradition rapportée dans les classiques, une implantation urbaine de quelque importance, qu'il s'agisse de la cité royale ou du plus petits des fiefs, avait pour centre architectural trois ensembles contigus : le palais du feudataire, le temple de ses ancêtres et l'autel du sol. Le Classique des poèmes rapporte ainsi l'œuvre des ancêtres de la dynastie Zhou:

Le plateau de Zhouyuan est une terre fertile, où le laiteron a la saveur de sucreries. Alors, vint le temps des discussions, le temps des délibérations. La tortue fut consultée. [Elle leur donna raison.] C'était le lieu, c'était le moment : c'était là qu'il fallait édifier les demeures [la nouvelle cité].

Chacun reçu son emplacement: qui à droite, qui à gauche.

Les bordures des champs furent tracées et la grille de leurs sillons alignée.

D'ouest en est, tout le monde s'affaira.

Les ministres des Travaux publics et de la Population furent chargés de la nouvelle édification.

On aligna au cordeau, on tassa la terre dans les coffrages des murs et le temple des ancêtres dressa [bientôt] sa silhouette majestueuse.

La terre s'entassait dans les paniers, les murs montaient dans les coffrages.

La terre fut tassée, [puis] les murs raclés et des centaines de murs édifiés plus vitc que le battement du grand tambour qui accompagnait les travaux.

On édifia [ainsi] la capitale aux portes imposantes; puis, le palais à l'entrée solennelle et majestueuse;

enfin, on éleva le grand autel du sol, lieu de ralliement de la population ${ }^{18}$.

Le temple des ancêtres et le temple du sol, pareils à deux sentinelles, encadraient le palais. Le premier de ces deux édifices préservait le témoignage des succès de la lignée, à commencer par le premier membre de la famille à avoir exercé une charge ou gagné un titre, et faisait ainsi office de garant de la légitimité de la position détenue par le clan dans la société. Le second représentait le territoire sur lequel s'étendait le pouvoir du souverain. Il était le symbole de l'autorité centrale et du lien vertical qui rattachait le feudataire à son prince et la population d'une localité donnée au gouvernement central : 
L'autel du sol servait aux anciens rois à faire éprouver la crainte de leur autorité et à transmettre leurs directives et leurs avertissements à leurs vassaux de manière à ce qu'ils acceptent docilement de servir leur chef ${ }^{19}$.

Ce devoir s'expliquait puisque l'autel du sol était aussi l'expression de l'entité administrative (localité) et des registres de ses habitants ${ }^{20}$; il représentait l'autorité charismatique de l'État et inspirait de ce fait de la crainte ${ }^{21}$. Les exécutions capitales avaient d'ailleurs lieu devant cet emblème du pouvoir suprême: «Qui m'obéit sera récompensé devant la tablette de mes ancêtres, qui me désobéit sera exécuté devant l'autel du sol ${ }^{22}$.» Dans la tradition classique, l'autel du sol n'est nullement considéré comme le symbole d'une communauté isolée: il est relié, tout comme le sol d'une localité, à un territoire plus large, le fief auquel il appartient et, comme lui, à un ensemble spatial plus vaste encore, le domaine de l'État. C'est ce qui explique que dans la tradition des Zhou de l'Est (-770/-221), transmise sous les Han, le personnage qui l'incarne n'est autre que l'arpenteur des neuf provinces, Yu le Grand lui-même ${ }^{23}$, et que les sacrifices offerts à l'autel du sol sont associés à ceux adressés aux quatre directions ${ }^{24}$, dont il constitue le point central (pour chaque communauté s'entend). Les prémices de cette paire de sacrifices se trouvent peut-être déjà dans deux inscriptions divinatoires:

Divination du jour wushen, devin Que: sacrifice aux quatre directions [sacrifice à l'autel du sol] (Yi 5272).

Victuaille à l'autel du sol et sacrifice di aux [directions] (Zhuihe 211) ${ }^{25}$.

Le talisman dit de Taiyi découvert à Mawangdui reflète, semble-t-il, une conception spatiale et politique similaire: un centre et quatre directions, mais aussi un centre et quatre frontières ${ }^{26}$. Le personnage central porte une coiffe de grue (à la manière des guerriers) et se trouve encadré par Leigong et Yushi, respectivement les dieux du tonnerre et de la pluie (protecteurs des armées). Il est dressé jambes écartées et surmonte trois dragons disposés en triangle. Il est entouré de part et d'autre de quatre personnages, porteurs pour trois d'entre eux d'une arme, et présentés chacun par un bref commentaire vertical. Le personnage central est Taiyi, l'étoile du «Grand un », Bêta de la Petite Ourse, considérée il y a deux millénaires comme «Étoile Polaire» (beichen), symbole du pouvoir central. Les quatre guerriers qui l'entourent sont accompagnés chacun d'une formule de protection contre les différents types d'armes. Les trois dragons qui forment le groupe de trois étoiles dit «fer de lance de Taiyi » (Taiyi feng) sont liés au processus de la forge des armes ${ }^{27}$. Enfin, sur le cœur de Taiyi, on peut lire entouré par un cercle le caractère she (autel du sol), dont la signification ne fait guère de doute. Les quatre guerriers peuvent sans grande difficulté être associés aux quatre directions de l'espace. Leur fonction de protection du centre constitué par Taiyi et l'autel du sol est implicite (voir illustration).

Les documents épigraphiques signalent que dès l'époque des Shang et des Zhou (ca. -1045/-221), la Chine connut la notion de territoires bornés par des 
limites et soumis, comme les populations qui les habitaient, à un pouvoir souverain, tel qu'il apparaît dans les inscriptions sur bronze des Zhou de l'Ouest (ca. -1045/-770):

Gloire au roi Wen qui reçu le grand mandat. Le roi Wu lui succéda et fonda notre État. [...] Il devint le détenteur de tout le territoire entre les quatre frontières et du pouvoir sur le peuple et ses fonctionnaires ${ }^{28}$.

La nature du pouvoir étatique décrit ci-dessus renvoie à la formule, présente dans plusieurs documents des Zhou de l'Est et attribuée par la tradition à l'empereur Shun, qui résume les fondements du système féodal : "Sous notre vaste ciel, tout le sol appartient au roi et tous ceux qui le foulent sont les sujets du roi ${ }^{29} »$; en d'autres mots, un territoire et une population, ou encore un sol, une force productive et son produit ${ }^{30}$. Le prince idéal serait celui qui, tel l'habile stratège décrit dans un passage du Guanzi, s'enquérrait d'abord de la surface des champs dont il dispose, puis du nombre des hommes qu'il peut entretenir, car qui dispose d'assez de surface de champs, disposera aussi de la population nécessaire ${ }^{31}$. Ainsi, la base de la bonne gestion de l'État semble bien être la maîtrise des potentialités du pays. Le système des fiefs s'organisait d'ailleurs principalement autour de deux types de dons: le territoire et la population qui y était fixée, éventuellement accompagnés du personnel administratif qui en avait la charge. L'une des rares inscriptions sur bronze détaillant l'attribution d'un fief à l'époque du roi Kang des Zhou (-1026/-1001), le célèbre vase de $\mathrm{Ze}$, nouveau marquis de Yi, décrit un territoire (peut-être situé dans la région de Suzhou), et cite un certain nombre de foyers de cultivateurs, ainsi que le personnel d'encadrement ${ }^{32}$ :

Le jour dingwei, au 4' mois, le roi consulte la carte de l'expédition des rois Wu et Cheng contre les Shang, puis celle des États de l'Est. Le roi se rend à Yi et là, devant l'autel du sol, tourné vers le sud, il annonce à $\mathrm{Ze}$, marquis de $\mathrm{Wu}$ : «Je déplace ton fief à Yi.» Dons: un vase de vin, une tablette de cérémonie en jade, un arc et cent flèches rouges, dix arcs et mille flèches noires. Dons de terre : rivières, trois cents ; [montagnes ?], cent et [?] ; agglomérations, trente-cinq; [routes ?] agglomérations de moindre importance ?], cent quarante. Don de personnel du roi en résidence à Yi : [soixante ?] sept $l i$ ${ }^{33}$, don de sept chefs de travaux et de [X centaines] cinquante travailleurs, don de six cents [?] six travailleurs ordinaires de Yi.

$\mathrm{Ze}$, marquis de $\mathrm{Yi}$, remercie le roi pour sa générosité [...] ${ }^{34}$.

\section{L'aménagement de l'espace}

\section{Repères identitaires: les neuf provinces}

Les dix zones dans lesquelles s'étaient établis depuis le néolithique une série de pôles culturels parfois inter-communiquants devaient finalement passer sous le contrôle de la plaine centrale à l'avènement de l'empire. Schématiquement leur distribution se fait comme suit: nord et ouest du Henan (1); sud du Shanxi (2); 
est du Henan et Shandong (3); Shaanxi et Gansu (4); ouest du Liaodong, nord du Hebei et région de Pékin (5); sud du Hebei (6); cours moyen du Yangzi (7); Jiangsu et Zhejiang (8); Anhui et Jiangxi (9); Sichuan (10) ${ }^{35}$.

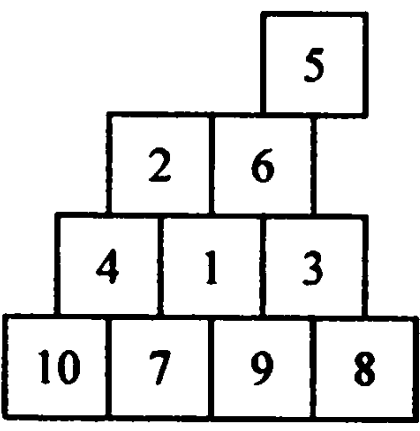

Ce découpage géographique s'inscrit dans un cadre mythique, celui des «traces des pas de $Y u »(Y u j i)$ dont la lignée est à l'origine de la première dynastie héréditaire des Xia (ca. -1900/-1570). Héros dompteur des eaux, Yu le Grand s'est vu attribuer, outre ses succès dans la lutte contre la grande inondation, l'aménagement du territoire des Xia, son relevé général et celui de ses montagnes et de ses rivières:

Immense [est l'espace qu'ont marqué] les traces des pas de Yu. 11 a tracé le carré des neuf provinces; il a ouvert le réseau des neuf routes. [Grâce à son œuvre], le peuple a habité au milieu des palais et des grands temples, le bétail a pâturé dans les herbages, chacun a trouvé sa place et $\mathbf{y}$ a demeuré ${ }^{36}$.

L'expression «les traces des pas de Yu» va progressivement et par extension signifier "le territoire de l'empire chinois», et désigne en fait le découpage géographique appelé les «neuf provinces (jiuzhou)». Plusieurs preuves épigraphiques démontrent l'existence de cette périphrase bien avant l'instauration de l'empire centralisé des Qin et des Han. L'inscription de l'aiguière (gui) du duc de Qin, déjà citée dans le recueil Planches archéologiques (Kaogu tu) des Song (960-1279), commence en ces termes:

Le duc de Qin dit: « Mon glorieux ancêtre a reçu le mandat du ciel et une demeure en dedans des traces de $\mathrm{Yu}{ }^{37}{ }^{37}$.

Shugong, un descendant de la famille princière de Song et donc de la lignée de la dynastie Shang ${ }^{38}$, associe également ses ancêtres à ce mythe:

Que sa gloire resplendisse, Cheng Tang [le fondateur de la dynastie Shang] est maintenant aux côtés de l'empereur d'en haut. Il a reçu le mandat du ciel et renversé la cité des Xia [...] avec l'aide de Yi Yin, son grand ministre qui l'assistait dans sa tâche. Il a établi son pouvoir sur [le carré] des neuf provinces et s'est installé dans l'ancienne cité de $\mathrm{Yu}^{39}$. 
Cet hommage implicite à la dynastie des Xia que les Shang avaient renversée démontre qu'à l'époque des Printemps et Automnes (-722/-481), le mythe associant Yu et les «neuf provinces» était déjà cité par les membres des maisons princières comme faisant partie de la connaissance commune. Wang Guowei (1877-1927) faisait déjà remarquer au début du siècle dernier que ces deux inscriptions infirmaient l'hypothèse, soutenue par Kang Youwei (1858-1927) et les membres les plus intransigeants de l'école «Doutes sur l'Antiquité» (Yigu pai), qui faisait de la notion des «neuf provinces» un mythe invente sous less Qin et les Han ou, à tout le moins, les Royaumes Combattants, afin de forger de toutes pièces le nouvel empire en formation. Si ces inscriptions démontrent l'antiquité de cette notion dans la conscience chinoise, elles ne donnent cependant pas d'informations sur le territoire qu'elle recouvre. La source la plus connue proposant un descriptif du territoire des Xia se trouve dans le Livre des Documents (Shangshu) et présente une nomenclature assez détaillée des neuf aires géographiques, de leurs reliefs et de leurs richesses. Le «Tribut de Yu» (Yu gong) - l'un des premiers textes géographiques de la Chine - apparaît comme un «vademecum » pour le prélèvement de l'impôt, il implique aussi leur appartenance à une entité politique commune dont l'idée se retrouve par exemple dans la formule « les neuf provinces ont toutes été ainsi pareillement réaménagées (jiuzhou youtong)». Une carte, citée dans le Mémoire sur les rites, résume les données géographiques du «Tribut de Yu»; elle est établie sur le modèle du carré des neuf cases, avec pour seul critère une mesure type de mille $l i$ :

Du mont Hengshan [du nord] au [à la partie méridionale du] Fleuve Jaune il y a près de mille $l i$; du Fleuve Jaune au Yangzi près de mille $l i$; du Yangzi au Hengshan [du sud] plus de mille $l i$; de l'est du Fleuve Jaune à la mer de l'Est plus de mille $l i$; de la région orientale du Fleuve Jaune à sa partie occidentale près de mille $l i$; de l'ouest du Fleuve Jaune au désert plus de mille li. À l'ouest [le territoire] s'arrête aux limites du désert et à l'est à la Mer orientale; au sud et au nord, aux deux monts Hengshan. [Ainsi] l'intérieur des quatre mers, en supprimant ce qui dépasse et en complétant les manques, correspond à un carré de trois mille $l i$ de côté, ce qui donne une surface de champs de XXX millions de $m u^{40}$.

Bien que le chapitre du «Tribut de Yu» précise que les «neuf provinces» forment un ensemble homogène, elles n'y sont pas présentées comme des juridictions administratives concrètes et aucune bordure ne vient les séparer, identiques en cela au schéma décrit ci-dessus ${ }^{41}$. À l'opposé des cartes de Mawangdui, ces textes n'ont nullement valeur de documents administratifs officiels, mais sont l'indice d'une unité culturelle, dans laquelle l'étendue des «neuf provinces» recouvre, tout comme les «traces des pas de Yu », l'ensemble du territoire «national » et font ainsi partie du bagage identitaire chinois. Reste à savoir si des repères géographiques précis sont associés à ces expressions. À ce sujet, il est intéressant de remarquer que la notion de «neuf provinces » semble avoir notablement évolué au cours des siècles, pouvant définir un territoire (de l'empire, d'un fief, etc.), 
mais aussi une juridiction administrative plus réduite à l'intérieur de celui-ci, pour désigner par exemple des quartiers de la capitale ${ }^{42}$. Comme le souligne un commentateur moderne, «les États se divisent en petits carrés de neuf provinces, mais s'insèrent à leur tour dans le grand carré des neufs provinces qui les contient tous ${ }^{43}$ ». On peut donc considérer que les «neuf provinces» de la tradition correspondaient à une grille d'organisation spatiale qui pouvait être appliquée à bien des territoires et qu'elles sous-tendaient la notion d'appartenance à un espace et à une identité définis.

Les textes classiques font référence à plusieurs complexes régionaux correspondant aux «neuf provinces». Selon certains, le centre de celles-ci se situerait aux alentours de l'actuelle Dengfeng, dans la région du mont Songshan (Henan) et ne comprendrait, d'après les reliefs cités, que le nord du Henan et le Shaanxi :

Le duc de Jin dit: «Notre État dispose d'importants atouts stratégiques. [...] Nous devons compter les atouts que représentent en particulier nos reliefs escarpés et le grand nombre de chevaux qu'on y élève.»

Son conseiller lui répondit: «Les quatre pics, les trois chemins, la cité de Yangcheng, la grande maison (dashi, autre nom de Songshan), la montagne Jingshan et le Zhongnan sont les principaux escarpements des neuf provinces et elles n'ont jamais été entre les mains d'un seul clan. Quand un des feudataires préposé à la garde de ces reliefs n'avait pas une vertu suffisante, son État disparaissait.

[Par ailleurs, rappelez vous que] le nord de la province de Ji (Jizhou), au sud duquel se trouve notre territoire, compte effectivement un grand nombre d'éleveurs de chevaux [en particulier parmi les populations de la steppe qui s'y sont fixées], mais n'a pourtant jamais constitué une entité politique indépendante. Ainsi peut-on constater aisément que les accidents du terrain et le nombre de chevaux dont on dispose ne sont nullement garants de la puissance d'un État ${ }^{44}$.

Dans ce modèle, la région de Jizhou, située au nord de la province du Shanxi et citée dans les "neuf provinces» du "Tribut de Yu», ne semble en effet pas s'inscrire dans les limites ici décrites. Dans d'autres sources, les «neuf provinces» paraissent d'ailleurs se limiter aux environs de Dengfeng:

Yu établit la cité de Yi au centre du Yinfang (Dengfeng). Il prit en charge l'administration de la région et les neuf provinces furent organisées ${ }^{45}$.

Dans les Discours des Royaumes, elles se situent toujours au Henan, mais plus au sud, et ne concerneraient que la région situé à l'ouest de la ville de Xie, proche de Nanyang (Henan):

Et au fait, qu'elle est la situation dans les neuf provinces à l'ouest de $\mathrm{Xie}^{46}$ ?

D'autres sources localiseraient le territoire ainsi désigné plus à l'est ${ }^{47}$. Il semblerait même que les "neuf provinces" de la région centrale (zhongguo) ${ }^{48}$ aient leur pendant dans des systèmes de «neuf provinces» existant chez les populations extérieures. En effet, le dernier chapitre du Zhuangzi explique que Yu 
le Grand ouvrit les cours du Fleuve Jaune et du Yangzi, permettant ainsi les communications avec «les neuf provinces des barbares des quatre directions» (siyi jiuzhou). Dans le Commentaire de Zuo Qiuming aux Printemps et Automnes, il est d'ailleurs fait mention d'une population extérieure appelée les «Rong de la région des neuf provinces" (jiuzhou zhi Rong). Cette appellation fait référence à un groupe de Rong qui avait émigré depuis le nord-ouest d'abord vers le nord du Henan, puis au nord du district de Songxian, toujours dans la même province, suite à une mésentente avec leur hôte, l'État de Jin ${ }^{49}$. Il est difficile de savoir si cette dénomination se réfère aux «neuf provinces» situées autour de Dengfeng, impliquant par là qu'ils avaient été acceptés par la population locale au point d'en faire partie, ou si elle concerne leur région d'origine, vraisemblablement située entre le Shaanxi et le Gansu.

Il est par ailleurs intéressant de noter que les traditions se rapportant aux anciens rois dépassent le cadre régional de la dynastie des Xia, et localisent une partie de leur action aux confins de ces territoires. Ainsi, si l'un des cinq souverains de la haute antiquité, Shun (r. ca. -2255/-2206), aurait régné sur la région située au sud-ouest du Shanxi, autour de l'actuelle Linfen, son action se prolonge bien plus au sud, car il serait mort lors d'une expédition contre les Miao qui auraient occupé la zone autour du lac Dongting (Hunan) ${ }^{50}$. Des remarques similaires peuvent être faites au sujet de Yu, le fondateur de la dynastie Xia, car selon la tradition il serait un «homme de l'Ouest», né parmi les Qiang de l'Oueśt ${ }^{51}$ et vraisemblablement originaire du nord du Sichuan. Aucune découverte n'est encore venue confirmer ces faits, mais il est possible de s'imaginer que cette légende antique reflète le souvenir d'importants mouvements migratoires, dont l'influence apparaît dans des pôles culturels couvrant une aire géographique allant du bassin du Sichuan au Henan. Bien qu'il ne soit pas permis de faire de trop rapides rapprochements, il est intéressant de remarquer que les récentes découvertes du site de Sanxingdui (Sichuan) attestent des liens étroits tissés entre les cultures de Shu (bassin du Sichuan) et celles de la plaine centrale. Les fouilles archéologiques commencent à livrer l'intéressante mosaïque des populations qui ont œuvré à une certaine «unification culturelle».

\section{Le domaine royal et les territoires extérieurs dans les inscriptions des Shang et des Zhou}

La tradition transmise par les classiques décrit ainsi l'établissement de la dynastie Shang et son système spatial:

L'empereur d'en haut ordonna à Cheng Tang le guerrier de rectifier le tracé des quatre frontières (sifang). Celui-ci nomma les princes qui régnèrent sur les neuf provinces et transmis le grand mandat à sa lignée et à Wuding, son descendant. [...] Le domaine royal est un carré de mille $l i$, dans lequel le peuple vient s'installer, mais les frontières du royaume (zhaoyu) sont aux confins des quatre mers ${ }^{52}$. 
L'intérieur des quatre mers est composé de neuf provinces, chacune formant un carré de mille li. Dans chaque province sont établis trente États (guo) de cent li de côté, soixante de soixante-dix $l i$ de côté, cent vingt de cinquante $l i$ de côté, en tout deux cent dix Etats. Les montagnes et les territoires des lacs et des marais ne sont pas attribués (feng). Le reste de la superficie est constitué de territoires annexes ou de champs intercalaires. Chacune des huit provinces contenait donc deux cent dix Etats [à l'exception du carré central qui était le domaine privé du fils du ciel] ${ }^{53}$.

La référence de base en ce qui concerne l'organisation territoriale sur laquelle s'étendait le pouvoir central des Shang consiste en une série d'inscriptions dont le modèle est le suivant :

Le jour yisi, le roi recourt à la divination en ce qui concerne la récolte de l'année à Shang.

Le roi annonce: «Faste»

Et quant à nos territoires de l'est?

Et quant à nos territoires du sud?

Et quant à nos territoires de l'ouest?

Et quant à nos territoires du nord ? (Cui 907) ${ }^{54}$

Les territoires des quatre directions cités ici correspondraient à l'ensemble de la superficie du royaume des Shang, dont l'étendue selon les récentes découvertes ne serait pas inférieure à celle que lui attribuent les classiques. Les fouilles archéologiques entreprises au siècle dernier apportent en effet déjà quelques concordances, telle par exemple la découverte à Hengyang (Hunan) de vaisselles de bronze Shang témoignant d'une grande maîtrise technique, et confirmant ainsi que la région du mont Heng [du sud], présentée dans le «Tribut de Yu» et le Mémoire sur les rites (cités plus haut) comme la limite méridionale des «neuf provinces ", était déjà entrée dans l'orbite de la plaine centrale à cette époque ${ }^{55}$.

Selon les estimations de Li Xueqin, et d'après les découvertes archéologiques entreprises sur l'ensemble du territoire chinois, les limites des dépendances du royaume, comme celles de l'inscription Cui 907 citée ci-dessus, engloberaient une zone s'étendant: a l'est jusqu'aux rivages de la mer; à l'ouest, à la partie occidentale du Shaanxi ; au sud, à la rive sud du Yangzi (Hunan et Jiangxi); au nord, à la partie centrale du Shanxi; au nord-est, à la partie ouest du Liaoning et au sudouest, aux limites du Sichuan ${ }^{56}$. Quant au statut des territoires inclus dans cet espace, nous ne disposons que de renseignements fragmentaires. Le contenu de cette inscription distingue un centre Shang, dit aussi dans des variantes plus tardives "la cité des Shang» (Shang), "la grande cité » (Dayishang) ou encore «la cité centrale de Shang» (Zhongshang) ${ }^{57}$, et quatre zones réparties en fonction des quatre directions qui lui sont rattachées. La demande de verdict divinatoire qui concerne ces zones implique à l'évidence un lien économique et politique entre leurs intérêts et ceux du pouvoir central. La notion de cité inclut par ailleurs, outre la capitale royale (Anyang), un territoire assez vaste, comme l'indiquent quelques inscriptions : 
- Le roi fait son entrée au nord de Shang. (Zhu 114.)

- Le roi fait son entrée et passe par Gong. (Nan ming 749.)

- Divination du jour dinghai : réponse donnée au lieu dit [X]. Le roi fait son entrée dans Shang la grande cité. (Yi 927.)

Les lieux dits Gong et $\mathrm{X}$ sont des territoires de chasse récurrents dans les inscriptions et se situeraient, d'après $\mathrm{Li}$ Xueqin ${ }^{58}$, sur la rive ouest du cours de la rivière Cishui (à l'est de Ciyang, Henan), à quelque $150 \mathrm{~km}$ de Anyang et donc entre cinq et dix jours de route à cette époque ${ }^{59}$. L'entrée dans la cité correspond donc au passage de la frontière d'un domaine royal dont les frontières se situeraient: au cours de la Cishui, au sud-ouest; à la chaîne du Taihangshan (Shanxi), au nord-ouest; au nord de l'actuelle ville de Shangqiu et, à l'est, dans la région située au nord et à l'ouest de Qufu. L'étendue de ce territoire correspond selon l'évaluation de Li Xueqin au carré de mille $l i$ de la tradition classique ${ }^{60}$.

La présence de ces pôles de culture Shang aussi éloignés entre eux ne signifie pas pour autant qu'ils aient été inclus à l'intérieur d'une ligne frontalière homogène, et ne préjuge pas non plus qu'ils aient été enclos dans des limites territoriales strictement définies. Certaines inscriptions oraculaires sous-entendent néanmoins leur existence. Ainsi, celles qui se rapportent aux visites et aux expéditions de chasse du roi, qui traversait fréquemment le territoire de l'un de ses vassaux en de telles occasions et y faisait même parfois défricher des champs par sa troupe et pour son propre profit :

- Divination du jour XX, verdict: donner ordre a $[X]$ d'entrer chez le marquis de Xian afin de préparer les champs. (Qianbian 6.14.6.)

- Divination effectuée par Bin le jour guisi, verdict: donner ordre à la troupe [...] de pénétrer dans l'État de Yangfang pour préparer les champs. (Jiabian 3510.)

Le terme "entrer» utilisé ici laisse entendre l'existence de frontières reconnues que le privilège royal permettait de franchir. Outre les feudataires du roi Shang, une multitude d'Etats indépendants entretenait avec la cour royale des relations parfois amicales, parfois nettement hostiles. La perspective d'une offensive extérieure ou d'un raid est souvent évoquée dans les inscriptions:

- Divination du jour renyin: cette année Fang fera-t-il une sortie? (Cuibian 1143.)

- Divination du jour shuyin: Fang fera-t-il une sortie? (Jieyun zanggui 1/4/3.)

Dans ce cas, c'est le terme «sortie » qui est généralement considéré comme une preuve de l'existence de limites reconnues, tout au moins par la cour royale, aux territoires des agresseurs.

La reconnaissance de telles frontières n'empêchait nullement la cour d'agrandir ses dépendances en récupérant à son profit les territoires indépendants qui n'étaient pas en mesure d'assurer leur propre défense. Cette pratique, encore en usage sous la dynastie Han ${ }^{61}$, consistant à emprunter des champs chez des voisins, pouvait, en particulier si ceux-ci étaient de petits États étrangers, se 
conclure par une annexion, comme l'explique une anecdote citée dans le Mengzi et qui témoigne sans doute de l'un des modes de développement du domaine royal des premières dynasties:

Cheng Tang résidait [dans sa ville] de Bo et Ge était son voisin. Le chef (bo) de Ge était de nature relâchée et n'accomplissait pas les sacrifices. Cheng Tang envoya auprès de lui un messager pour lui en demander la raison et il répondit: «Je n'ai pas de bétail.»Cheng Tang lui fournit bœufs et moutons que le chef de Ge mangea sans toujours accomplir de sacrifices. Tang dépêcha à nouveau un messager pour lui en demander la raison et il répondit: «Je n'ai pas de céréales. » Cheng Tang ordonna alors aux troupes de Bo d'aller labourer son territoire [et] les vieux et les enfants eurent [ainsi] à manger. Le chef de $\mathrm{Ge}$ voulu dépouiller sa population du vin et de la nourriture qu'elle détenait, ceux qui refusaient de lui donner les libations étaient exécutés. Un enfant mangeait un plat de céréales accompagné de viande et Ge le fit tuer pour lui voler sa nourriture. [...] Au nom de ce crime, Cheng Tang lança contre lui une expédition militaire et tout le monde dans l'empire dit [alors]: «ll ne recherche pas les richesses de l'empire, mais seulement à venger un père et une mère " ${ }^{62}$.

Quant aux relations de la cour royale avec ses ennemis, elles ont toutes les caractéristiques des relations d'État à État ${ }^{63}$. Ainsi, par exemple, celles que la cour entretenait avec le pays de Gong ${ }^{64}$ :

Divination: faut-il ordonner d'aller faire une expédition de reconnaissance au Gongfang? (Jin 12-17.)

Ce pays était en effet en mesure de lever une armée et de soutenir une guerre de trois ans en étant soumis à une pression militaire de plusieurs milliers d'hommes, de même qu'il pouvait prendre la tête d'une expédition menée conjointement avec les troupes d'un autre Etat hostile. Un certain nombre d'inscriptions (dont plusieurs datant du règne de Wuding) révèlent les inquiétudes des rois Shang au sujet de la sécurité de leurs territoires frontaliers:

Jour guisi, divination effectuée par Que [première période du règne Wuding]:

Décade sans catastrophes?

Le roi consulte les sorts et dit: un présage néfaste est apparu. Le danger se dirige vers nous.

Le cinquième jour suivant, jour dingmao, le danger arrive. De l'ouest, Wu de Zhi vient annoncer: le pays de Tufang a attaqué notre campagne est (bi) et a endommagé deux de nos agglomérations. Le pays de Gongfang a également fait une incursion à notre frontière ouest ${ }^{65}$.

On retrouve la distinction entre domaine royal et fiefs extérieurs dans les inscriptions des Zhou de l'Ouest, parmi lesquelles celle du tripode Lingfang distingue nettement le personnel administratif du domaine royal appelé ici «les cent fonctionnaires " (baixing) et les chefs de fiefs en charge des quatre frontières (sifang) ${ }^{66}$, les fonctionnaires de l'extérieur (waifu): 
À l'audience royale du matin à Chengzhou, des ordres ont été transmis aux responsables des trois offices, au grand secrétaire, aux ministres, aux directeurs des îlots murés et aux responsables des cent artisans ainsi qu'aux feudataires des trois degrés hou, zi et nan afin qu'ils les transmettent aux quatre frontières ${ }^{67}$.

Le terme «quatre frontières » tel qu'il est utilisé dans les inscriptions des Zhou de l'Ouest désigne les fiefs qui se trouvent au-delà du domaine royal et à l'intérieur des frontières extérieures reconnues du territoire. Une organisation analogue se trouve dans l'inscription du «Grand tripode de Yu » (Da Yu ding) datant de la 23e année du règne du roi Kang (-1026/-1001), dans laquelle l'un des termes actuellement les plus courants pour désigner les frontières d'un territoire, bian, est déjà utilisé :

J'ai entendu dire que les feudataires des trois degrés (hou, tian et nan) qui protègent les frontières (bian) de Yin et les cent fonctionnaires du domaine royal avaient tous sombré dans l'ivrognerie et que c'est ainsi que la dynastie perdit son armée ${ }^{68}$.

Le terme «bian» accolé aux trois rangs des feudataires indique que les fonctions de ceux-ci étaient bien en relation avec le sens originel du titre de hou - gardien des frontières, des postes avancés ou éclaireur, appellation militaire encore en usage sous la dynastie Han pour le caractère proche hou ${ }^{69}$. La notion des «quatre frontières » citée ici est analogue à celle que lui donne Zheng Xuan (127-200) dans ses notes aux Rites des Zhou, où il explique qu'elle correspond à l'ensemble des feudataires en titre (zhuhou) ${ }^{\text {no }}$. Le même auteur, dans ses annotations au Livre des Odes (Shijing), donne un sens encore plus large et définit les «quatre frontières ( (sifang) comme étant «l'ensemble des branches du peuple des $\mathrm{Xia}^{71}$ ».

Si l'on considère l'inscription du plat de Xi Jia, datant du règne du roi Xuan (-827/-782), ces deux définitions apparaissent restrictives, car le domaine des barbares soumis depuis un certain temps est associé à celui des quatre frontières:

Le roi ordonne à [Xi Jia] de prendre la charge de responsable des finances [et des réserves] de la cité de Chengzhou [Luoyi] et de ses quatre frontières, y compris ( $z h i$ yu) les barbares Yi du sud de la Huai qui nous payent l'impôt depuis les temps anciens. Qu'ils n'osent pas refuser de payer la soie, les réserves et la force de travail [qu' ils nous doivent]. Qu'ils n'osent pas refuser que leurs biens affluent vers les camps de nos armées [?] et vers nos marchés. En cas de désobéissance, des représailles de l'armée sont prévues en châtiment. Quant à nos feudataires (zhuhou) et à nos fonctionnaires (baixing), qu'ils n'osent pas non plus refuser de remplir nos marchés et qu'ils ne tentent pas de s'y livrer à des trafics illégaux. En cas d'infraction, des châtiments sont prévus. Xi Bo Jifu en souvenir a fait fondre ce plat ${ }^{72}$.

L'utilisation du terme «zhi yu» (jusque) paraît inclure à l'intérieur des «quatre frontières », outre les cent fonctionnaires du domaine royal et les feudataires, une autre catégorie, les peuples tributaires. Les territoires de ces derniers et des peuples non soumis étaient vraisemblablement définis. Une frontière et une ville 
centrale $(d u)$, telle est la description la plus concise d'un État hostile suffisamment important pour être cité dans une inscription cérémonielle:

Le roi s'efforce d'imiter les rois Wen et Wu quant au gouvernement du territoire et de ses limites. [L'un des] chefs des États du sud a osé violer notre territoire. Le roi a organisé l'entrée dans ses frontières et l'attaque de sa cité. Leur [...] chef a envoyé un émissaire devant le roi. Les barbares du sud et ceux de l'est sont venus en audience, vingt-six États en tout [...]. Que Fu vive dix mille années et qu'il protège les quatre frontières ${ }^{73}$.

Ainsi, d'après tout ce qui précède, la Chine connut dès les Shang et les Zhou la notion de territoires bornés par des limites et l'on peut inférer que le territoire des Huaxia était séparé de celui des barbares, afin que selon la célèbre formule de Confucius, citée dans le Commentaire de Zuo aux Printemps et Automnes et reprise sans cesse pendant les périodes de troubles et d'invasion qui marquèrent maintes fois l'histoire de Chine, «Les étrangers des bordures $(y i)$ ne forment pas de projets contre le territoire des Xia, et les barbares Yi ne viennent pas semer le désordre dans le peuple des $\mathrm{Hua}^{74}{ }^{\text {». }}$

\section{Les zones d'allégeance et la représentation des frontières de l'État}

Un passage du "Tribut de Yu» présente en un schéma simplifié la hiérarchie des devoirs des vassaux vis-à-vis de leur suzerain, et donc de l'influence du pouvoir des Xia sur les différents rangs des feudataires et les populations qui reconnaissaient leur autorité. Le carré de ce modèle, découpé en bandes concentriques organisées autour et à partir de la capitale, a une superficie de $5000 \mathrm{li}$ de côté:

Le domaine royal (dianfu) s'étend à $500 \mathrm{li}$ autour de la capitale [...]. De sa bordure extérieure jusqu'à 500 li plus loin se trouve le territoire des marquis (houfu) [...]. 500 li plus loin encore était fixée la bordure extérieure du territoire des " vassaux " (suifu); 500 li plus loin: bordure extérieure du territoire des vassaux par alliance (yaofu); 500 li plus loin enfin : région celle des vassaux des steppes (huangfu) ${ }^{75}$.

Un tel déploiement en rayons des territoires correspond au devoir d'allégeance au pouvoir central des différentes populations, devoir qui décroît au fur et à mesure de leur éloignement du centre. Il distingue le domaine royal, directement géré par la cour, de celui confié aux feudataires (territoires des marquis) et marque, indirectement, la frontière du territoire du royaume des Xia. Dans le Mémoire des rites, on trouve un modèle simplifié:

L'intérieur du carré de 1000 li [autour de la capitale] était appelé "domaine royal» (dian). Les domaines situés au-delà de ce carré de $1000 \mathrm{li}$ constituaient les «territoires d'apanage» (cai) et les «terres d'exil» (liu) ${ }^{76}$. 
Comme le fait remarquer $\mathrm{Gu}$ Jiegang, les cinq zones d'allégeance correspondent aux cinq catégories de sujets, ce que montre très clairement un passage du Discours des royaumes commentant le modèle du Mémoire des rites: les hauts fonctionnaires du domaine royal, ou feudataires de l'intérieur (bangnei), devaient pourvoir aux offrandes quotidiennes; les marquis, ou feudataires de l'extérieur (bangwai), aux offrandes mensuelles; les gardiens des marches, ou vassaux invités (binfu), aux offrandes saisonnières; les Man et les Yi, ou les populations alliées (yaofu), fournissaient un tribut annuel, et les Rong et les $\mathrm{Di}$, ou les peuples des steppes (huangfu), se présentaient une fois par génération a la cour afin de faire acte d'allégeance 7 . Il y a ainsi trois sortes de domaines: celui relevant directement de la cour, les territoires des feudataires (bangwai et binfu) et ceux des peuples des frontières (yaofu et huangfu).

Avant l'organisation d'un empire au sens moderne avec un appareil répressif couvrant toute la superficie du territoire et capable de mener avec succès des expéditions lointaines, l'influence de la cour semble, $d$ 'après les textes classiques, avoir été fondée sur un degré d'indulgence qui augmentait avec l'éloignement du centre $^{78}$. Le mot d'ordre le plus représentatif du style du pouvoir ancien s'exprimerait par la formule «souplesse envers les populations lointaines, générosité envers les populations proches " (rouyuan neng'er) ${ }^{79}$. Une devise que l'on trouve dans une inscription sur bronze des Zhou de l'Ouest, le grand tripode de $\operatorname{Ke}(D a$ $\mathrm{Ke}$ ding, $\mathrm{Ix}^{\mathrm{e}}$ siècle avant notre ère):

Oh, Shihua! Mon noble ancêtre! Quelle n'était pas la subtilité de son esprit! Ne méditait-il pas ses plans avec un calme souverain ! Oui, vraiment, éclatante fut sa vertu ! Ainsi sut-il servir le roi Kongwang, établir l'ordre dans sa maison, faire ruisseler sur le peuple les effets de sa générosité et montrer souplesse envers les populations lointaines, indulgence avec les populations proches (rouyuan neng'er). Ainsi, il n'oublia jamais la volonté du ciel et fut considéré comme loyal par chacun dans l'empire ${ }^{80}$.

Cette formule constitue la leçon que les hommes politiques postérieurs ont tirée de la grande expédition de Shun contre lcs Miao (citée plus haut), même si elle pourrait avoir été bien plus difficile que ne le laisse entendre la tradition, car il trouva la mort dans les régions du sud lors de ces opérations. Quoiqu'il en soit, l'expédition est ainsi décrite:

À l'époque de Shun les Miao refusaient de se soumettre. Yu se préparait à les attaquer, mais Shun le retint: «Ne pas avoir une vertu suffisante et recourir aux armes, voilà qui va à l'encontre de la voie.» Pendant trois ans, Shun se contenta de leur transmettre ses enseignements et d'organiser des danses avec le bouclier et la hache. Au bout de ces trois années, les Miao se soumirent ${ }^{81}$.

Le domaine sur lequel vivaient les souverains ne cessera de s'étendre suite à l'augmentation régulière de leurs dépenses, ce qui mettra parfois à mal le respect de ce modèle hiérarchique, obligeant la cour à trouver de nouveaux emplacements suivant des critères similaires. Le choix de la nouvelle capitale des Zhou de 
l'Ouest à Luo (act. Luoyang), tel qu'il est décrit dans les Mémoires historiques, en constitue un bon exemple: située au centre du territoire relevant de la cour des Zhou, Luo «permettait d'accorder les distances de toutes les régions de l'empire dans toutes les directions et de leur attribuer un nombre de li uniforme, afin d'y échelonner aisément les impôts ${ }^{82}$ ", décidés en fonction de l'éloignement du territoire de chaque vassal de la capitale. Les zones d'influence ainsi établies courent sur une distance de $\mathbf{2 5 0 0 ~ l i ~ d e ~ p a r t ~ e t ~ d ' a u t r e ~ d e ~ l a ~ c a p i t a l e ~ p o u r ~ f o r m e r ~}$ un carré de 5000 li et paraissent correspondre assez justement au territoire des trois dynasties de l'antiquité. Il est encore fait référence à ce modèle dans plusieurs fragments de textes des Zhou de l'Est, tel par exemple un passage de l'Art de la guerre de Sunzi qui contient une petite théorie des fondements du système féodal:

Sun Wu dit: «Les empereurs [de la haute Antiquité] et les rois [des trois premières dynasties] demeuraient à l'intérieur des quatre mers, au centre du territoire de $5000 \mathrm{li}$. Comment auraient-ils eu les moyens de monopoliser à leur seul avantage toutes les productions et tous les profits d'une telle superficie? Aussi firent-ils des parts de ce territoire et les attribuèrent-ils à des vassaux, afin que chacun d'eux puisse tirer profit des richesses de la région qui lui avait été attribuée, manger le produit de son propre sol et mettre à contribution les forces de sa propre population. Ainsi [empereurs et rois] n'eurent-ils pas à déplorer que l'excessive superficie de leur territoire les empêchât de le gouverner» ${ }^{83}$.

Aux vassaux des territoires extérieurs, il n'était demandé qu'un service minime bien qu'important, puisqu'ils constituaient le bouclier extérieur du territoire, comme le montre le célèbre passage du Commentaire de Zuo aux Printemps et Automnes vantant les méthodes de soutien mutuel sur lesquelles se fonde une bonne défense du territoire:

Le premier ministre de Chu fit fortifier la cité de Yin, la capitale de l'État. L'administrateur [de la cité de Shen] commenta ainsi ce fait: «L'édification de cette enceinte ne servira à rien. [...] Ces murailles ne pourront être défendues. Jadis la défense du fils du ciel était l'affaire des barbares des quatre directions. Puis quand le pouvoir central déclina sa défense ne reposa plus que sur ses feudataires. La défense du territoire d'un feudataire tenait jadis à ses quatre voisins. Mais quand le pouvoir des feudataires déclina, leur seule ligne de défense fut alors leurs quatre frontières. Il vaut mieux en cas de tension nouer des traités d'assistance réciproque dans les quatre directions et $[\ldots]$ veiller à ce que son peuple vive en paix dans ses campagnes, sans crainte au-dedans, ni ennemi au-dehors; les murailles des cités ne servent alors à rien. Il faut rectifier les bordures des parcelles (jiangyi), distribuer rationnellement les terres et les champs [...], veiller à ce que tous les groupes de cinq aient un chef (wuhou), établir des relations de confiance avec ses voisins [...] et l'on est dès lors en mesure de se premunir contre tout raid inattendu ${ }^{84}$.

Certaines inscriptions des Zhou décrivent des scènes de bonne entente entre le roi et ses feudataires, ou la colère du souverain à la suite d'une trahison. 
L'inscription du chaudron du marquis Yu Fang de E, probablement datée de l'époque du roi $Y i$, rapporte les détails de l'accueil que le marquis fit au souverain lorsque celui-ci traversa son territoire à la suite d'une expédition punitive dans le sud:

Sur le chemin du retour le roi a fait halte à Bei [?]. Là, le marquis de $\mathrm{E}$ a offert au roi un vin de cérémonie. Le roi lui a répondu en lui dédiant une nouvelle tournée de vin. Le marquis a remercié le roi. Le roi a donné le signal de la fin du banquet. Un concours de tir a l'arc a suivi. Yu Fang et le roi ont tous deux pris part au tir. Yu Fang a atteint la cible. Le roi l'a félicité. Une nouvelle tournée de vin a été offerte. Le roi a fait don à Yufang de cinq paires de pièces de jade, de quatre chevaux et de cinq faisceaux de cent flèches. Yu Fang a salué les mains jointes en frappant le sol du front. Il remercie le fils du ciel de sa générosité et fait fondre ce chaudron pour que ses enfants et ses pctitsenfants le conservent comme un trésor précieux pendant dix mille années ${ }^{85}$.

Le fief de $\mathrm{E}$ se trouve dans une région limitrophe des territoires étrangers, sur les frontières sud-est du royaume, et le marquis semble avoir noué des liens matrimoniaux avec la famille royale ${ }^{86}$. Une seconde inscription présente un contexte beaucoup moins idyllique que la première, et l'on apprend que le même marquis s'est rebellé:

Hélas le ciel envoie un grand malheur à notre État. Yu Fang, marquis de E, s'est rebellé. Il a pris la tête des barbares du sud de la Huai et de ceux de l'est pour lancer une offensive générale contre nos États du sud. Ils sont arrivés jusqu'à [?].

Le roi ordonna aux armées de l'ouest et aux huit armées de la cité de Yin de le punir:

Châtiez Yu Fang marquis de E et n'épargnez ni les vieillards, ni les enfants. [...] Yu a pris la tête des chars et des fantassins de Wugong. Il est arrivé devant E, l'a attaqué et a fait prisonnier Yu Fang, prince de cet État.

L'événement en question paraît lié aux troubles du règne du roi Yi qui sonnaient le déclin de la dynastie des Zhou de l'Ouest, et dont les Mémoires historiques conservent peut-être une trace:

A l'époque du roi Yi des Zhou, la maison royale était affaiblie. Les feudataires ne venaient plus en audience. Ils se livraient mutuellement à de multiples exactions. Xiong Qu ayant gagné la confiance des populations du Fleuve et de la Han leva ses armées et descendit pour les châtier. [...] Il avança ainsi [vers le sud] jusqu'à $E^{87}$.

La répression menée par Xiong Qu s'acheva par l'installation d'un nouveau marquis à E, et la cité de E devint la nouvelle capitale de l'État de Chu, tandis que Danyang, l'ancienne capitale, fut dès lors connue sous le nom de E de l'Ouest. 


\section{Le tombeau de Shun et les frontières de l'empire}

Dans la tradition, l'extension des frontières n'est pas sans relation avec le souvenir des derniers grands souverains de l'Antiquité dont les règnes précédèrent la fondation de la première dynastie héréditaire des Xia. Ainsi, Wudi des Han accomplit le sacrifice aux montagnes pour les mânes de Shun au Mont Jiuyi. Le mont Jiuyi est situé au nord-ouest du district de Jianghua (Hunan): il marquait à l'époque des Han la limite de l'orbite culturelle des Huaxia et constituait un relief stratégique important ${ }^{88}$. L'une des deux cartes trouvées à Mawangdui, celle dite «des reliefs de terrain », témoigne de l'importance de ce site et confirme la poussée chinoise vers le sud (carte 1). L'autre décrit les dispositifs de défense d'une région soumise à une forte pression militaire de la part du royaume méridional de Yue (carte 2). La première donne le relevé d'un vaste territoire qui s'étend jusqu'à la mer, représentée sur le côté supérieur, et s'étire jusqu'à l'«intérieur des frontières de l'ennemi» (fengzhong) au sud. Sur le tombeau de Shun, indiqué par deux caractères (Shun di), se dressent orgueilleusement les silhouettes des neuf stèles - la plus grande étant au centre. Il est vraisemblable que ce lieu chargé d'histoire ait représenté la pointe avancée de la civilisation Huaxia aux confins des frontières méridionales.

Quant à la ligne frontière elle-même, elle est signalée sur la carte par un tracé droit, allant d'est en ouest. Cette ligne passe à intervalles rapprochés par un certain nombre de cols alignés d'est en ouest et entourés d'un dispositif de petits postes de garde. Ces passes, figurées par une stylisation du caractère shan, suivent le tracé de la chaîne de montagnes et semblent correspondre à un tracé plus ou moins linéaire qui pourrait être la frontière méridionale du territoire de la dynastie des Han. Le nom de chacun des cols se termine par le caractère feng et pourrait désigner une borne frontière. La ligne continue perpendiculairement du sud au nord, indiquant qu'un dispositif semblable la distingue des deux zones administratives contiguës. Le tracé de cette ligne n'a nullement force de document administratif: les deux cartes de Mawangdui sont visiblement opportunistes et marquent probablement l'équilibre des forces à la suite des avancées chinoises vers le sud. Il n'est nullement impensable que la simplicité d'un tracé graphique géométriquement régulier, sur le modele d'une ligne horizontale et semblable en cela aux modèles carrés cités plus haut, ait été reproduite sur le terrain avec les adaptations nécessaires aux accidents du relief (fleuves, chaînes de montagne etc.) qui pouvaient constituer un appui tactique pour la défense de la frontière.

Trois lignes de trois et quatre garnisons d'armée dans leurs cantonnements murés échelonnés du sud au nord, viennent garder cette ligne frontière sur toute sa largeur et sous trois axes nord-sud différents. Les commentateurs chinois voient dans ce dispositif une organisation de trois forces de premier, de second et de troisième échelon, distribuées autour d'un quartier général de forme triangulaire et dont les tourelles de défense sont représentées sur un plan à l'extérieur des 


\section{Paola Calanca}

murailles. Une distance d'environ trente $l i$, équivalant à une journée de marche d'une armée, sépare chaque échelon. Le modèle n'est pas sans évoquer, au niveau opérationnel, l'organisation des formations militaires de l'époque des Zhou de l'Est. Une série d'îlots est représentée en divers points de la carte avec la mention de la population disponible pour de futurs enrôlements. Quelques-uns sont accompagnés de la mention «ici $\mathrm{X}$ nombre de familles», mais la majorité porte seulement les formules «vide» ou «population non encore revenue », ce qui laisse entendre que la région pourrait avoir été dévastée lors d'opérations militaires et qu'une grande partie de la population s'était dispersée sur les routes pour fuir la zone des combats.

Ainsi les modèles contenus dans les Rites des Zhou et transmis par les textes classiques, du carré central du domaine royal entouré à distance variable par les domaines des feudataires, puis par ceux des pays voisins dont les liens avec la cour se distendaient au fur et à mesure de leur éloignement - à l'image d'une construction géométrique et lexicale -, ne semblent pas être démentis par la réalité de l'époque, telle que l'histoire nous permet de la reconstituer ${ }^{89}$. Par ailleurs, s'il n'est pas encore possible de retracer, documents à l'appui, l'histoire des frontières de la Chine antique, l'existence de la notion de frontière et de son marquage ne fait pas de doute, même si le tracé des limites ne peut être aujourd'hui esquissé et fut modifié à de multiples reprises. Ajoutons encore, quant au territoire de l'empire unifié, que ses limites étaient vraisemblablement marquées par des signes visibles, et ce même sur sa frontière méridionale, où les faibles dangers représentés par les populations non soumises et la luxuriance du territoire ne nécessitaient pas de dispositif aussi élaboré et complet que celui des fortins et des tours de guet de la grande muraille.

\section{Notes}

* Il arrive parfois que l'on s'éloigne de son domaine de recherche, en l'occurrence l'histoire de la défense maritime Ming et Qing, et qu'on s'initie à d'autres périodes historiques. Bien qu'une telle démarche comporte des risques certains, elle ne répond pas moins à une nécessité de chercheur. Travaillant sur les textes militaires de la fin de l'empire, très empreints de la tradition classique, j'ai été amenée à m'intéresser à l'époque ancienne. Il est évident que je ne me serais pas lancée seule dans cette aventure et ce travail doit beaucoup à François Wildt, qui a savamment su m'ouvrir au monde de l'Antiquité et m'initier aux sources anciennes. S'agissant d'un délit d'initiée, cet article est avant tout basé sur les sources textuelles, dont la lecture doit évidemment se poursuivre. À l'avenir, il faudra également s'intéresser à l'ensemble des sources archéologiques traitant des frontières. J'adresse mes remerciements également à $\mathrm{M}$. Hans Van Ess qui a relu la première mouture de cet article et aux autres patients lecteurs. Si des erreurs et des imprécisions persistent, elles me sont entièrement imputables. 
1. Huainan zi jishi, vol. 2, j. 8, p. 563. Ce passage est extrait du chapitre «La chaîne originelle » du Maître de Huainan, ouvrage remontant au Ir $^{\leftarrow}$ siècle avant notre ère. En français, cette œuvre a été traduite et annotée sous la direction de Charles le Blanc et Rémi Mathieu (Gallimard, "Bibliothèque de la Pléiade», Philosophes taoïstes, II, Paris, 2003) et le passage ici cité se trouve, avec quelques variantes, à la page 334 .

2. Tan Sitong, Ren xue, 1998, p. 235.

3. Liji zhengyi, j. 21, p. 1414. Pour une traduction française de cet ouvrage, voir Mémoires sur les bienséances et les cérémonies, trad. Séraphin Couvreur, Cathasia, Paris, 1950 (ce passage se trouve p. 498).

4. Shiji, j. 128, p. 3233.

5. Zhongguo da baike quanshu, «Kaogu xue», p. 34. Les fouilles de ce site ont été menées par l'Institut d'Archéologie de l'Académie des Sciences de Chine entre 1954 et 1957.

6. Liji zhengyi, j. 21, p. 1418.

7. Yuhai, j. 140, p. 2613.

8. Guanzi jiaozhu, j. 5, p. 85.

9. Shuowen jiezi, j. 13 inf., p. 286.

10. A ce sujet, voir par exemple Lü shi chunqiu, j. 3, p. 638.

11. Xunzi jijie, j. 6,p. 179.

12. «Le ciel est un, la terre deux; le ciel est trois, la terre quatre (Zhouyi zhengyi, j. 7, p. 81).

13. Xunzi jijie, j. 17,p. 449-450. Une formulation analogue se trouve également dans un passage du Maître de Huainan: "Ce que l'on appelle le "Un" est ce qui n'a pas de pair dans l'empire et se tient unique et solitaire. En haut, il entre en relation avec les neuf parties du ciel et en bas avec les neuf carrés de la terre.» (Huainan zi jishi, j. 1, p. 58, ou dans la traduction de C. le Blanc et R. Mathieu p. 32.)

14. Liutao, j. 3, p. 466. Cet ouvrage fait partie des Sept Classiques militaires et sa composition remonte à l'époque des Royaumes Combattants.

15. On le trouve, par exemple, ainsi explicité dans un des traités de stratégie de la fin des Royaumes Combattants: «Dans le camp, l'armée du centre, celles de gauche, de droite, d'avant et d'arrière, ont chacune un carré de terrain pour se disposer. Il sera entoure par des murets qui ne devront pas être franchis sans laissez-passer." (Wei Liao zi, j. 2, p. 398-399.)

16. Shuihudi Qin mu zhujian, p. 116. Ce document a été découvert en 1975-1976 dans une tombe située dans la province du Hubei, dans le district de Yunmeng, au nord du lac Dongting, au sud de l'ancienne capitale du pays de Chu. Les plaquettes en bambou datent de la fin du urr siècle avant notre ère et correspondent à dix ouvrages ayant été rédigés entre la fin des Royaumes Combattants et le début des Qin, dont une partie du code de cette dernière dynastie.

17. On peut considérer cette «pierre dressée » comme une forme archaïque d'autel du sol, puisque le caractère le désignant apparaît également dans les inscriptions avec la même fonction:

Divination du jour wushen, devin Huan : une grande cité sera-t-elle établie à Tang? (He 40352.)

Question: une grande cité sera-t-elle établie à l'autel du sol (tu) de Tang? (He 40353, face ; aussi notée Jin 611.) 
Le terme tu de cette inscription est en général compris she, à savoir l'autel de la terre (Chen Mengjia, Yinxu buci zongshu, 2004, p. 584).

18. Mao shi zhengyi, j. 16/2, p. 509-512.

19. Chunqqiu Gongyang zhuan zhushu, j. 24, p. 2347. Au sujet de ce lien vertical: «Suivant les lois des Zhou, l'édification d'un autel du sol était obligatoire pour tous les groupements de cent familles au moins. Depuis les Qin et les Han, tout groupe à partir de vingt-cinq foyers est tenu d'édifier un autel du sol.» (Liji zhengyi, j. 25, p. 1449.)

20. Shiji, j. 47, p. 1932.

21. Les Entretiens de Confucius expliquent d'ailleurs que «Les Zhou plantaient leurs autels du sol de châtaigniers (li), afin que le peuple tremble (li) » (Lunyu zhengyi, j. 4, p. 119).

22. Shangshu zhengyi, j. 7, p. 155. La découverte en 1965, à Xuzhou (Jiangsu), sur le site de Qiuwan à Tongshan, d'un autel de pierre datant de la fin des Shang, analogue aux autels du sol fait en pierre, cités précédemment, et que le Huainan zi attribue spécifiquement à cette dynastie, confirme cette réputation (Huainan zi jishi, j. 11, p. 1254). L'autel constitué de quatre pierres dressées était situé au centre d'un petit groupe d'habitations, de telle sorte que les habitants pouvaient toujours l'avoir sous les yeux lors de leurs activités. À proximité, vingt squelettes et deux crânes humains, ainsi que douze squelettes de chiens furent exhumés (Yu Weizhao, Xian Qin liang Han kaoguxue lunji, 1985, p. 54-58).

23. Chunqiu Zuozhuan zhengyi, 29॰ année du règne du duc Zhao de Lu, j. 53, p. 2124 : «Le fils de Gonggong avait pour nom Goulong, il devint le prince du sol. » Ou encore, dans les Mémoires sur les rites: «Le fils de Gonggong fit régner l'ordre dans les neuf provinces, aussi lui dédia-t-on des offrandes en tant qu'autel du sol.» (Liji zhengyi, j. 46, p. 1590.) Le Zuozhuan est un des commentaires aux Annales de la Principauté de Lu (-722/-484), composé par Zuo Qiuming (époque des Printemps et Automnes).

24. Mao shi zhengyi, j. 18/1, p. 552: «J'apporte les céréales dans le vase à offrande et aussi un agneau au pelage uni. Ils sont pour l'autel du sol. Ils sont pour le sacrifice aux quatre directions pour que nos champs aient une récolte abondante.»

25. Yu Xingwu, Jiagu wenzi gulin, 1979, p. 186.

26. C'est le lien qu'établissent implicitement les Rites des Zhou, dans les attributions du ministère de la Terre, entre l'autel du sol d'un territoire et ses bordures: «Grand ministre de la population. Il veille aux tracés des limites du domaine royal et de celles des domaines annexes, fait creuser les fossés qui les longent et les fait planter d'arbres. Il fait établir les banquettes de l'autel du sol et de celui des céréales et les fait planter d'arbres, en fonction de l'essence régionale et établit ainsi le nom de l'autel du groupe d'habitations afférent et de la campagne périphérique [...].» (Zhouli zhengyi, j. 18, p. 689-692.) L'érection du tertre de l'autel du sol est ici complémentaire des bornes des quatre frontières du domaine dont il représente le centre. Un même lien est établi dans le paragraphe traitant des tâches du « responsable des bornes de terre» (fengren), rattaché au ministère de la Terre: «Le responsable des bornes de terre établit l'autel du sol du roi et fait élever les murets de terre qui l'entourent sur ses quatre côtés. Il supervise l'organisation du tracé du domaine royal qu'il fait planter d'arbres. Quant un fief est institué, le responsable des bornes fait élever les murets de terre qui entourent ses autels du sol et des céréales, et fait également établir 
les limites de ses quatre frontières (feng qi si jiang). Quant à l'établissement d'une ville secondaire et de sa campagne périphérique, il en va de même» (Zhouli zhengyi, j. 22, p. 890-891). Le commentaire des Han précise: «Le domaine royal est un carré de mille li de côté; ses frontières (feng) sont les tracés de bordures (jie) actuelles.»

27. Li Ling, Zhongguo fangshu xu kao, 2000, p. 207-238.

28. Inscription dite du «Grand tripode de $\mathrm{Yu}$ » (Da Yu ding), datée du règne du roi Kang (-1026/-1001), voir Guo Moruo, Liang Zhou jinwenci daxi tulu kaoshi (ci-après Daxi lu), $\mathrm{n}^{\circ}$ 18, 1958 ; Chen Mengjia, Xi Zhou tongqi duandai, 2004, p. 100-104.

29. Mao shi zhengyi, j. 13/1, p. 463. Une formule que l'on retrouve par exemple aussi dans Mao shi zhengyi, j. 13/1, p. 463; Mengzi zhengyi, j. 18, p. 637. Un sol et des céréales, tel se présente le destin inévitable qui enferme l'homme dans un statut de sujet de l'État, où même le produit des terres non cultivées appartient au souverain, et qui ne laisse donc à celui qui refuse de manger les céréales que lui accorde le gouvernement que le choix de se laisser mourir de faim. C'est ce que rappelle la légende de Boyi et Shuqi qui refusèrent de servir les Zhou par fidélité aux Shang (Shiji, j. 61, p. 2121-2124). Une anecdote citée dans les «Biographies des immortels», collectées par Ge Hong (283-343 ou 363), constitue a contrario une exception trop rare pour ne pas confirmer la règle susdite: «Le vieux maître des bords du fleuve [...] habitait une hutte de roseaux sur les rives du fleuve Jaune. L'empereur Wen [des Han] aimait l'étude de Laozi, mais avait quelques difficultés en certains passages [du texte] que personne ne pouvait lui expliquer. [...] Il entendit parler du commentaire que le vieillard avait rédigé et dépêcha un messager pour lui poser ses questions. [...] Le vieux répondit: "La voie et la vertu sont choses trop honorables pour être expliquées à distance." L'empereur se rendit alors en personne devant sa hutte et réitéra poliment ses questions. [Il dit]: "Sous l'immensité du ciel il n'est terre qui ne soit terre du roi et tous ceux qui foulent cette terre sont de même sujets du roi. [...] Vous détenez le dao, mais n'en êtes pas moins l'un de mes sujets." [...] Le vieillard plaça alors ses paumes l'une dans l'autre, s'assit et monta dans les airs à plusieurs toises de hauteur et répondit d'en haut en penchant la tête: "Je ne monte pas jusqu'au ciel, je ne suis pas lié par les hommes et ne suis pas obligé de demeurer sur la terre. Je ne suis donc le sujet de personne." L'empereur salua front contre terre et dit: "Veuillez m'excuser".»( Shuoku, j. 3.)

30. Le fonctionnement optimal d'un tel dispositif apparaît, par exemple, dans un document datant probablement des Zhou de l'Ouest, où l'on voit le contrôleur des champs diriger non seulement sa brigade de paysans, mais aussi les travaux du champ de mille $m u$ auxquels participaient toute la cour et le fils du ciel en personne: "Le contrôleur des champs donne ordre aux fonctionnaires d'organiser le travail des champs [...] et annonce à sa brigade: "Au travail! Lors de la grande cérémonie du labour des mille mu du champ royal [qui vient d'avoir lieu], le directeur des champs a donné l'exemple, suivi par [mes] assistants, puis par [leurs] assistants; ensuite, [ce fut le tour] du ministre des travaux publics, suivi par le ministre de la population, puis [par ordre de succession] le précepteur royal, le mâtre d'armes du roi, le grand secrétaire, le responsable du temple des ancêtres et, enfin, le roi s'est mis à son tour au travail et ils ont fait de même pour le sarclage." Le peuple très impressionné se consacre alors avec ardeur aux labours et défriche les limites de ses champs, se courbant jour après jour sur sa bêche, travaillant sans éprouver de fatigue. Ainsi les 
biens ne font pas défaut et une bonne atmosphère de coopération règne au sein de la population.» (Guoyu, j. 1/6, p. 15-21.)

31. Guanzi jiaozhu, j. 53, p. 1025. L'historien des institutions Du You (735-812), citant le Guanzi, réaffirme cette évidence: «Celui qui veut organiser son État doit d'abord savoir de combien d'hommes il dispose et pour savoir de combien d'hommes il dispose, il faut d'abord connaître la surface des terres en sa possession.» (Tongdian. j. 3, p. 55.)

32. Li Xueqin, Zhouchu yigu shidai, 1997, p. 260-263.

33. La construction «x dizaines... you... $x$ unités » utilisée ici n'étant employée dans les inscriptions que pour les numérations superieures à cinquante et un $l i$ ancien (îlot d'habitation urbain ou rural) et regroupant en moyenne de vingt-cinq à cinquante familles, il est possible de se faire une idée du nombre de la population donnée lors de l'attribution du fief: soixante-sept $l i$ (nombre minimum) équivalent ainsi à mille sept cent cinquante ou trois mille trois cents familles, et ce uniquement pour le personnel directement lié à la cour (Li Xueqin, 1997, p. 255).

34. Chen Mengjia, Xi Zhou tongqi duandai, p. 14-15. Le releve scrupuleux de la population ainsi attribuée laisse entendre que les livrets de famille, que l'on appellera «livrets d'inscription à l'autel du sol » (de la localité), étaient peut-être déjà en usage. Les termes employés dans les inscriptions oraculaires pour désigner certaines troupes de fantassins (dengren), littéralement «conscrits», impliquent l'existence de «rôles» d'inscription et de levées pour des campagnes militaires : «Divination du jour bingwu, devin X: levée de trois mille conscrits pour attaquer le Gongfang» (Jialing 156); "Verdict: levée de cinq mille conscrits pour une mission de reconnaissance au Gongfang» (Xu 1, 13, 15).

35. Li Ling, Li Ling zi xuanji, 1997, p. 53-55. Dans cet ouvrage, ces zones sont mises en relations avec les différents foyers culturels néolithiques, les dynasties historiques et les États des Zhou de l'Est.

36. Chunqiu Zuozhuan zhengyi, $4^{e}$ année du duc Xiang de Lu, j. 29, p. 1933.

37. Gu Jiegang, Gushi bian, vol. 7,p. 361-367. Le Kaogu tu, dont la préface date de 1092, a été compilé par Lủ Dalin (1046-1092), qui y classe et date quelque deux cents pièces de bronze (des Shang aux Han) et une dizaine de jade.

38. L'État de Song avait été attribué aux descendants des Shang par leurs successeurs de la dynastie des Zhou et était situé dans l'actuelle province du Henan; il disparaîtra au III siècle avant notre ère.

39. Inscription de la cloche dite de Shugong datant du règne du duc Zhuang de Qi (-553/-548). Voir à ce sujet Li Ling, 1997, p. 75.

40. Liji zhengyi, j. 13, p. 1347. Pour une autre traduction de ce passage, voir Mémoires sur les bienséances et les cérémonies, p. 321-322.

41. La description des frontières dans le «Tribut de Yu» est d'ailleurs très sommaire: «Quant aux limites, elles vont à l'est jusqu'à la mer, à l'ouest jusqu'au désert et s'arrêtent quelque part au nord et au sud.» (Jin Jingfang et Lü Shaogang, «Shangshu Yu Xia shu» xinjie, p. 432.) Cette lecture paraît plus satisfaisante que l'interprétation classique: «et s'arrêtent au nord et au sud jusqu'où portent les directives du roi ». (Shangshu zhengyi, j. 6, p. 153; Mémoires sur les bienséances et les cérémonies, p. 88-89.) 
42. À ce sujet, voir Lü Simian, lorsqu'il cite parmi les tâches attribuées à l'arpenteur dans les Rites des Zhou celle de diviser la capitale en neuf quartiers (jiuzhou), suggérant ainsi qu'il s'agissait d'une organisation de l'espace qui pouvait être appliquée à n'importe quelle surface (Lü Simian dushu zhaji, 1986, p. 423).

43. Jin Jingfang et Lü Shaogang, « Shangshu Yu Xia shu » xinjie, 1996, p. 292.

44. Chunqiu Zuozhuan zhengyi, $4^{*}$ année du règne du duc Zhao de Lu, j. 42, p. 2033. Cette conversation intervient entre le duc de Jin (région du sud Shanxi et du nord Henan) et l'un de ses conseillers, et laisse entendre qu'à l'époque des Printemps et Automnes, les territoires désignés sous l'appellation des neuf provinces pouvaient encore être multiples.

45. Mozi jiangu, j. 2, p. 47.

46. Guoyu, j. 16, p. 514-515. D'après les notes, la ville de Xie était la capitale du fief de Shen, conféré au début des Zhou à la lignée des Qiang, alliés du clan Ji, fondateur des Zhou. La région, protégée par deux barrières montagneuses, constitue un site stratégique pour contenir la poussée des voisins méridionaux, les Man (populations extérieures). Elle fut ensuite l'objet de fréquentes offensives lancées par l'État de Chu, qui allait devenir la principale menace contre la plaine centrale pendant toute la période des Printemps et Automnes. La donation du fief a été célébrée dans le Livre des Odes (Mao shi zhengyi, j. 18/3, p. 565-568).

47. Huainan zi, j. 4, p. 311-313, ou dans la traduction de C. le Blanc et R. Mathieu p. 161162.

48. Lü Simian, 1986, p. 72. Lü Simian cite en effet un commentaire du chapitre "Almanach saisonnier » du Huainan zi où il est dit que, grâce à des remblais élevés dans les cours d'eau, Yu organisa les «neuf provinces» de l'État de la région centrale (zhongguo jiuzhou).

49. Chunqiu Zuozhuan, $17^{\circ}$ année du duc Zhao de $\mathrm{Lu}$, j. 48, p. $1574 ; 22^{\bullet}$ année du duc Zhao de Lu, j. 22, p. 1644.

50. «La tombe de Shun se trouve dans la campagne de Cangwu» (Liji zhengyi, j. 7, p. 1281). La montagne Cangwu est située dans la région de Ningyuan (Hunan). Au sujet de l'identification de ces endroits, voir Yu Weizhao, 1985, p. 235-238.

51. Shiji, vol. 2, j. 15, p. 686.

52. Mao shi zhengyi, j. 20-3, p. 623.

53. Liji zhengyi,j. 11, p. 1323. Couvreur, p. 268.

54. Hu Houxuan, Jiaguxue Shangshi luncong chuji, 1989, p. 279.

55. Wenwu kaogu gongzuo sanshi nian, p. 311-312.

56. Li Xueqin, Yindai dili jianlun, 1959.

57. Chen Mengjia cite encore d'autres variantes (Yinxu buci zongshu, p. 255).

58. Li Xueqin, 1959, p. 95-96.

59. Yang Shengnan, Jiaguxue yu Yin Shang shi, 1983, p. 140-141.

60. Li Xueqin, Dangdai xuezhe zixuan wenku, 1998, p. 112.

61. C'est le cas, par exemple, du pays de Shanshan, dont le territoire est sablonneux, salin, et ne comporte que peu de champs cultivables. Les travaux de culture pour sa consommation en céréales sont ainsi effectués chez les voisins, à qui il achète également le grain comme l'indique le commentaire de Yan Shigu (581-645) (Han shu, vol. 12, j. 96, p. 3876). Shanshan est le nom que prit le royaume de Loulan (Xinjiang actuel) en 77 avant notre ère. Il est situé au sud-ouest du lac Lop Nor, à 
$6100 l i$ à l'ouest de Chang'an; il comptait, à l'époque des Han, 1570 foyers, une population de 14100 personnes, et disposait d'une armée de 2912 hommes. Dans le même chapitre du Han shu, consacré aux frontières occidentales (Xiyu), il est précisé que d'autres pays sont aussi obligés d'emprunter des champs à leurs voisins, comme Puli (act. Xinjiang) qui cultive à Shache, ou encore Yinai à Shule et Shache (p. 3883).

62. Mengzi zhengyi, j. 12, p. 431-434.

63. Pour de plus amples informations au sujet des relations entretenues entre les Shang et les Etats limitrophes, voir Chen Mengjia, Yinxu buci zongshu, p. 249-312 et Li Xueqin, 1959; en anglais, Nicola Di Cosmo, «The Northern Frontier in pre-imperial China », p. 885-966.

64. Le pays de Gongfang devait se situer au nord-ouest du territoire des Shang, quelque part au nord du Shaanxi et du Shanxi, ou même dans la région de l'Ordos. D'après les nombreuses mentions de son nom dans les inscriptions divinatoires, ses contacts avec les Shang étaient fréquents, en particulier sous le règne de Wuding, lorsque de nombreuses campagnes furent lancées contre ce pays. À son sujet, voir Nicola Di Cosmo, «The Northern Frontier in pre-imperial China», p. 907-908; Edward L. Shaughnessy, «Historical Geography and the Extent of Early Chinese Kingdoms », 1989, p. 1-13.

65. Guo Moruo, Buci tongzuan, 1983, p. 438. Le pays de Tufang est, de même que celui de Gongfang, un nom qui revient souvent dans les inscriptions divinatoires du règne Wuding des Shang (Chen Mengjia, Yinxu buci zongshu, p. 272).

66. Le caractère fang est la représentation graphique d'un instrument aratoire. Dans les inscriptions oraculaires, il apparaît souvent précédé par le nom d'une ethnie et désigne alors un pays indépendant ou un territoire reconnu comme vassal par la cour des Shang. Le terme sifang peut également désigner les quatre directions de l'espace: «Infini comme les quatre directions de l'espace que nulle frontière ne vient arrêter.» (Zhuangzi, j. 6, p. 51.) Le caractère fang étant proche phonétiquement et graphiquement de pang, dans son acception de "côté», les sifang peuvent ainsi représenter les quatre territoires situés dans les quatre directions à l'extérieur d'un domaine, tout comme ses quatre frontières, c'est-à-dire les quatre côtés du carré figurant son territoire. L'expression s'applique en particulier aux «quatre frontières» du territoire du royaume ou de l'empire, comme dans la célebre «Chanson du grand vent» du fondateur de la dynastie Han: «Mais où trouverai-je des héros pour garder mes quatre frontières?» (Shiji, 1996, vol. 2, j. 8, p. 389.)

67. Inscription du vase rituel $\mathrm{Yi}$ (Lingfang yi) datant du règne du roi Cheng (1063-1027 avant notre ère), voir Guo Moruo, Daxi lu 3 ; Chen Mengjia, Xi Zhou tongqi duandai, p. $35-40$.

68. Guo Moruo, Daxi lu 18; Chen Mengjia, Xi Zhou tongqi duandai, p. 100-104.

69. Graphiquement, le caractère hou désigne une «flèche» et peut-être une «cible» (hou). Selon Chen Mengjia (Yinxu buci zongshu, p. 328), son sens premier est analogue à celui de son quasi homonyme hou, variante non distinguée graphiquement dans l'écriture ancienne, signifiant encore dans la langue tardive «éclaireur», ou "gardien des frontières », suivant la définition donnée dans le Shuowen (p. 165, sup.): épier, surveiller (siwang).

70. Zhouli zhengyi, j. 64, p. 2698.

71. Mao shi zhengyi, j. 17/4, p. 548. 
72. Inscription du plat de Xi Jia (Xi Jia pan), voir Guo Moruo, Daxi lu 134, Chen Mengjia, Xi Zhou tongqi duandai, p. 323-326.

73. Inscription de la «cloche de Fu» (Fu zhong), dite aussi «cloche de Zongzhou », datant du règne du roi Xuan (827-842 av. notre ère), voir Guo Moruo, Daxi lu 25; Chen Mengjia, Xi Zhou tongqi duandai, p. 310-313.

74. Chunqiu Zuozhuan zhengyi, 10 année du règne du duc Ding de Lu, j. 56, p. 2148.

75. Shangshu zhengyi,j. 6, p. 153. Couvreur, p. 87-88.

76. Liji zhengyi, p. 1325. Couvreur, p. 271.

77. Guoyu, j. 1, p. 4 ; Gu Jiegang, Shilin zashi, p. 4.

78. Shiji, j. 6, p. 236.

79. Mao shi zhengyi, j. 17/4, p. 548; Chunqiu Zuozhuan zhengyi, $10^{\circ}$ année du règne du duc Ding de Lu, j. 56, p. 2147-2148.

80. Inscription du grand tripode de $\mathrm{Ke}$ (Da Ke ding) datant du roi Yi (-869/-858) ou du roi Li (-857/-842), voir Guo Moruo, Daxi lu, p. 110-111; Chen Mengjia, Xi Zhou tongqi duandai, p. 260-263.

81. Han Feizi jijie, j. 19, p. 445.

82. Shiji, j. 4, p. 133.

83. Taiping yulan, j.626.

84. Chunqiu zuozhuan: $23^{\circ}$ année du règne du duc Zhao de Lu, j. 50, p. 2022-2023.

85. Chen Mengjia, Xi Zhou tongqi duandai, p. 216-219.

86. Chen Mengjia, Xi Zhou tongqi duandai, p. 217.

87. Shiji, j. 40, p. 1692.

88. Huaninan $z i, j .18$, p. 1288-1289 : «Qin shi huang envoya cinq armées et cinq cent mille hommes [...] garder les passes stratégiques du sud. La seconde armée pris position sur le relief stratégique de Jiuyi.»

89. Hans van Ess, Politik und Gelehrsamkeit in der Zeit der Han : Die Alttext/ NeutextKontroverse, 1993, p. 132-137. 


\section{BibLIOGRAPHIE}

\section{Sources primaires}

Chunqiu Gongyang zhuan zhushu (Commentaire de Gongyang à la Chronique des Printemps et Automnes), Beijing Daxue chubanshe, «Shisan jing zhushu», vol. 2122, Pékin, 2000.

Erya, Beijing Daxue chubanshe, «Shisan jing zhushu», vol. 24, Pékin, 2000.

Guanzi jiaozhu (Commentaire au Guanzi), Zhonghua shuju, Pékin, 2004.

Guo Moruo, Buci tongzuan (Recherches sur les inscriptions oraculaires), Kexue chubanshe, Pékin, 1982.

Guo Moruo, Liang Zhou jinwenci daxi tulu kaoshi (Édition illustrée et commentée des inscriptions sur bronze de la dynastie des Zhou), Kexue chubanshe, Pékin, 1983.

Guoyu (Discours des Royaumes), Shanghai guji chubanshe, Shanghai, 1998.

Han Feizi jijie (Commentaires au Han Fei zi), Zhonghua shuju, Pékin, 1998.

Han shu (Histoire des Han), Zhonghua shuju, Pékin, 2002.

Huainan zi jishi (Commentaires au Maître de Huainan), Zhonghua shuju, Pékin, 1998.

Liji shijie (Commentaire au Mémoire sur les rites), Zhonghua shuju, «Zhongguo gudian mingzhu shizhu congshu », Pékin, 2001, 2 vol.

Liutao (Six Fourreaux), Jiefangjun chubanshe, «Zhongguo bingshu jicheng», 1, Shenyang, 1987.

Lunyu zhengyi (Commentaires Entretiens), Zhonghua shuju, Pékin.

Lü shi chunqiu (Les Printemps et Automnes de Maître Lii), Guji chubanshe, «Ershi'er zi », Shanghai, 1986.

Mawangdui Han mu boshu (Les livres sur soie de la tombe Han de Mawangdui), Wenwu chubanshe, Pékin, 1980.

Mao shi zhengyi (Commentaire du Livre des Odes de Mao), Beijing Daxue chubanshe, «Shisan jing zhushu », vol. 4-6, Pékin, 2000.

Shangshu zhengyi (Commentaire au Livre des documents), Zhonghua shuju, «Shisan jing zhushu », Pékin, 2003, vol. 1, p. 109-258.

Shiji (Mémoires historiques), Sima Qian, Zhonghua shuju, Pékin, 1996.

Shuowen jiezi (Dictionnaire étymologique), Zhonghua shuju, Pékin, 1998.

Sunzi (Maître Sun, traité de stratégie), Jiefangjun chubanshe, «Zhongguo bingshu jicheng », vol. 1, Shenyang, Beijing, 1990, p. 1-34.

Shuihudi Qin mu zhujian (Les plaquettes en bambou d'une tombe Qin de Shuihudi), Wenwu chubanshe, Pékin, 2001.

Shuoku (Réservoir des anecdotes), Zhejiang guji chubanshe, Hangzhou, 1986.

Taiping Yulan (Encyclopédie de l'ère Taiping, supervisée par l'empereur), Li Fang et al., Zhonghua shuju, Pékin, 1963.

TaN Sitong, Ren xue (Étude sur la vertu d'humanité), Zhongzhou guji chubanshe, 1998.

Tang liu shuyi yizhu (Le Code des Tang et ses commentaires), Zhonghua shuju, Pékin, 1984.

Tongdian (Somme des textes classiques), Du You, Zhonghua shuju, Pékin, 2003.

Wei Liao zi (Maître Wei Liao, traité de stratégie), Jiefangjun chubanshe, «Zhongguo bingshu jicheng », 1, Shenyang, Beijing, 1990.

Xunzi jijie (Commentaires du Xunzi), Zhonghua shuju, Pékin, 1988.

Yantie lun (Discussion sur le sel et le fer), Zhonghua shuju, Pékin, 1996. 
Yili zhushu (Mémoire sur les bienséances), Beijing Daxue chubanshe, «Shisan jing zhushu », vol. 10-11, Pékin, 2002.

Yi Zhou shu huijiao jizhu (Commentaires au Livre perdu des Zhou), Shanghai guji chubanshe, Shanghai, 1995.

Yuhai (Océan de jade), Jiangsu guji chubanshe, Nankin, 1987.

Zhouli zhengyi (Commentaires aux Rites des Zhou), Shangwu yinshuguan, Shanghai, 1938.

Zhouyi zhengyi (Le sens correct des Mutations des Zhou), Beijing Daxue chubanshe, «Shisan jing zhushu, vol. 1, Pékin, 2000.

Zhuangzi, Shanghai guji chubanshe, «Ershi er zi», Shanghai, 1986.

\section{Sources secondaires}

Chen Mengjia, Xi Zhou tongqi duandai (Périodisation des bronzes des Zhou de l'Ouest), Zhonghua shuju, Pékin, 2004.

CHEN Mengjia, Yinxu buci zongshu (Synthèse au sujet des inscriptions oraculaires des Yin), Zhonghua shuju, Pékin, 2004.

CORDELL D. K. Yee, «Reinterpreting Traditional Chinese geographical Maps», dans Cartography in the Traditional East and Southeast Asian Societies, Harley J.B. et Woodward David (éd.), The University of Chicago Press, Chicago et Londres, p. 35 70.

Di Cosmo Nicola, «The Northern Frontier in pre-imperial China », dans Cambridge History of Ancient China, Michael Loewe et Edward L. Shaughnessy (éd.), Cambridge University Press, Cambridge, 1999, p. 885-966.

Ess Hans van, Politik und Gelehrsamkeit in der Zeit der Han: Die Alttext/ NeutextKontroverse, Harrassowitz, Wiesbaden, 1993.

Ess Hans van, Von Ch'eng I zu Chu Hsi. Die Lehre vom Rechten Weg in der Überlieferung der Familie Hu, Harrassowitz, Wiesbaden, 2003.

Falkenhausen Lothar von, «Issues in Western Zhou Studies: A review Article», Early China, 18, 1993, p. 139-226.

FALKenHAuSEN Lothar von, «The Waning of the Bronze Age: Material Culture and Social Developments 770-481 B.C.», dans Michael Loewe et Edward L. Shaughnessy (éd.), The Cambridge History of Ancient China, Cambridge University Press, Cambridge, 1999, p. 450-544.

Gu Jiegang, Gushi bian (Débats sur l'histoire ancienne), Shanghai guji chubanshe, Shanghai, 1982.

Gu Jiegang, Shilin zashi chubian (La forêt de l'histoire), Zhonghua shuju, Pékin, 1963.

Harley J.B., Woodward David (éd.), Cartography in the Traditional East and Southeast Asian Society, The University of Chicago Press, Chicago et Londres, 1994.

Hu Houxuan, Jiaguxue Shangshi luncong chuji (Recueil d'essais d'histoire Shang d'après l'étude des inscriptions divinatoire), Hebei jiaoyu chubanshe, Shijiazhuang, 1989.

JIN Jingfang et LO Shaogang, "Shangshu yu Xia shu» xinjie (Nouveaux éclaircissements sur les «documents de la dynastie Xia» du Livre des Documents), Liaoning guji chubanshe, Shenyang, 1996.

LI Ling, Zhongguo fangshu kao (Essais sur l'art divinatoire et médical chinois), Renmin Zhongguo chubanshe, Pékin, 1993. 
Li Ling, Zhongguo fangshu xu kao (Essais sur l'art divinatoire et médical chinois, suite), Dongfang chubanshe, Pékin, 2000.

LI Ling, $Z i$ xuanji (Li Ling par lui-même), Guangxi Shifan daxue chubanshe, Guilin, 1997.

LI Xueqin, Dangdai xuezhe zixuan wenku, Li Xueqin juan (Les lettrés contemporains par eux-mêmes, volume de Li Xueqin), Anhui Jiaoyu chubanshe, Hefei, 1998.

Li Xueqin, Yindai dili jianlun (Le territoire à l'époque des Yin), Kexue chubanshe, Pékin, 1959.

Li Xueqin, Zouchu yigu shidai (Au-delà de l'école du scepticisme historique), Liaoning Daxue chubanshe, Shenyang, 1997.

LiU Xiang, Chen Kang, Shang-Zhou guwenzi duben (Manuel pour les anciens caractères des Shang et des Zhou), Yuwen chubanshe, Pékin, 1989.

Lo Simian, Lü Simian dushu zaji (Notes en lisant l'histoire de M. Lü Simian), Shanghai guji chubanshe, 1982.

PINES Yuri, «The search for Stability: Late Ch'un-ch'iu Thinkers », Asia Major, 10, 1997, p. 1-47.

Pines Yuri, " The One That Pervades the All" in Ancient Chinese Political Thought: the Origins of "The Great Unity" Paradigm », T'oung Pao LXXXVI, n' 4-5, 2000, p. 280324.

ShaUGHNESSY Edward L., "Historical Geography and the Extent of Early Chinese Kingdoms », Asia Major, 2-2, 3e série, 1989, p. 1-22.

YANG Kuan, Xi Zhou shi (Histoire des Zhou de l'Ouest), Shanghai renmin chubanshe, Shanghai, 1999.

YANG Shengnan, Jiaguxue yu Yin Shang shi (L'étude des inscriptions divinatoires et l'histoire des dynasties Yin et Shang), Shanghai guji chubanshe, Shanghai, 1983.

Yu Weizhao, Xian Qin liang Han kaoguxue lunji (Essais sur l'archéologie des Qin et des Han), Wenwu chubanshe, Pékin, 1985.

Yu Xingwu (éd.), Jiagu wenzi gulin (Recueil de commentaires sur les caractères des inscriptions oraculaires), Zhonghua shuju, Pékin, 1979.

Zhongguo da baike quanshu (La grande encyclopédie chinoise), «Kaogu xue » (Archéologie), Zhongguo da baike quanshu chubanshe, Pékin et Shanghai, 1986.

Zhongguo gudai ditu ji (Recueil de cartes anciennes chinoises), Cao Wanru et al. (éd.), Wenwu chubanshe, Pékin, 1990. 


\section{Glossaire}

\begin{tabular}{|c|c|}
\hline Anyang 安陽 & Huashan 華山 \\
\hline baixing 百姓 & Huaxia 華复 \\
\hline Ban Gu 班固 & Huai 淮 \\
\hline banglei 邦内 & huangfu 荒服 \\
\hline bangwai 邦外 & Ji流 \\
\hline beichen 北辰 & Ji 紀 \\
\hline binfiu 㨐服 & Jizhou䒚州 \\
\hline Bo 毫 & jiangyi 㽬埸 \\
\hline bo 伯 & jiao 刘 \\
\hline Boyi 伯夷 & jie 界 \\
\hline cai 采 & Jin \\
\hline Cangwu 荅梧 & Jingshan 荊山 \\
\hline chen lie 陳列 & Jiuyi 九知 \\
\hline Cheng Tang 成湯 & jivshow 九州 \\
\hline Cheng wang 成王 & jiuzhou youlong 九州敒闹 \\
\hline Chengzhou 成周 & jiuzhow zhi Rong 九州之找 \\
\hline Da Ke ding 大克鼎 & Kang wang 康王 \\
\hline Da Yu ding 大孟鼎 & Kang Youwei 康有為 \\
\hline dashi 大室 & Kong Yingda 孔穎䞗 \\
\hline datong 大同 & Leigong 雷公 \\
\hline Dayishang 大邑商 & $l i$ 傈 \\
\hline Dengfeng 登封 & $l i$ 栗 \\
\hline dengren 等人 & Liyun 禮運 \\
\hline di 地 & Lingfang yi 令方 \\
\hline dian 甸 & liu 流 \\
\hline dianfu 甸服 & Lu 麻 \\
\hline Dongting 洞庭 & Luoyi 洛㫕 \\
\hline Du You 杜佑 & Mawangdui 馬王堆 \\
\hline$d n$ 都 & Man 玮 \\
\hline Erlitou二里頭 & Miao 苗 \\
\hline fang 方 & nan 男 \\
\hline feng 封 & Nanyang 南陽 \\
\hline feng qi si jiang 封其四疆 & Ningyuan 要速 \\
\hline fengjiang 封疆 & Puli 蒲犁 \\
\hline $\mathrm{Ge}$ 莺 & Qi 弯 \\
\hline Ge Hong 葛洪 & Qiang 美 \\
\hline Gonggong 共I & Qin秦 \\
\hline Goulong 句龍 & Rong 戎 \\
\hline gioo 圆 & ronyuan neng 'er 秀遠能用 \\
\hline Hengshan 恒小 & Sanxingdui 兰星堆 \\
\hline Hengshan 衡山 & Shache 涉車 \\
\hline Hengyang 衡陽 & Shanshan 部善 (pays) \\
\hline how 侯 & Shang 商 \\
\hline hou 猴 & she 社 \\
\hline houfu侯服 & Shen 沈 \\
\hline Hua 茟 & Shu 蜀 \\
\hline
\end{tabular}


Paola Calanca

Shule 疏勒

Shuqi 叔齐

Shuihudi 睡虎地

Shun 舞

Shun di 舞帝

sifang 四方

siyi jiuzhon 四夷九州

Song 末

Songshan 禽山

Songxian 满縣

suifu 綏服

Taiyi feng 太一鋒

Tan Sitong 譚開同

III土

Tufang 土方

wan wu葛物

Wang Guowei 王四維

Wang Yinglin 王應锞

Wei 魏

Wei 渭

Wuding 武丁

wuhou伍候

Xi Bo Jifu 兮的吉父

Xi Jia pan 兮甲船

$X i y$ 西域

Xia 夏

Xie 㴬

Xiong Qu 熊染

Yan Shigu 顏師古

yaofu 要服

Yi 夷

Yi 宜 (marquis de)

$\mathrm{Yi}$ 益

$y i$ 筒

yi 更

yigu pai 疑古派

yiren 一人

Yinai 依耐

Yin $尹$

Yinfang 陰放

Yu gong 禹頁

Yuhai 玉海

Yuji 禹跡

Yushi 雨師

ynambian 缘㟫

Yue 越

Ze 决

zhaoyu望域 zhen 略

sheng feng jiang iF.封堛

Zheng Xuan 矨玄

Zhi ist:

zhiyu 至丁

zhongguo 中國

Zhongnan 中南

Zhongshang 中商

Zhou 周

Zhou 紂

zhon 洲

Zhouyuan 周原

zhuhou 諸侯

Zongzhou 宗周

Zuo Qiuming 左丘明 
L'aménagement du territoire et la notion de frontière à l'époque ancienne

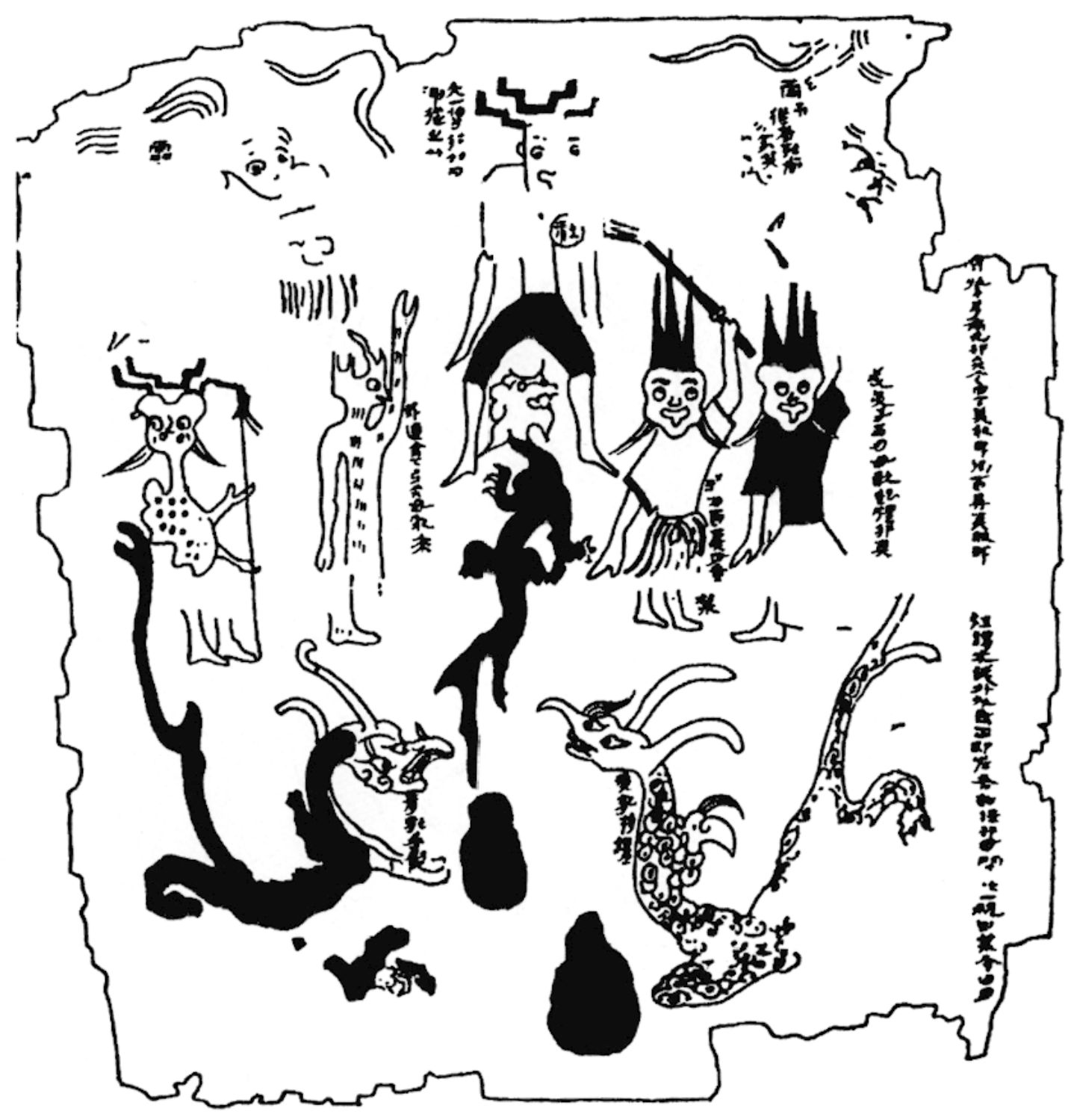

Taiyi (Mawangdui, d'après Li Ling, 2000, p. 224). (D.R.) 
Paola Calanca

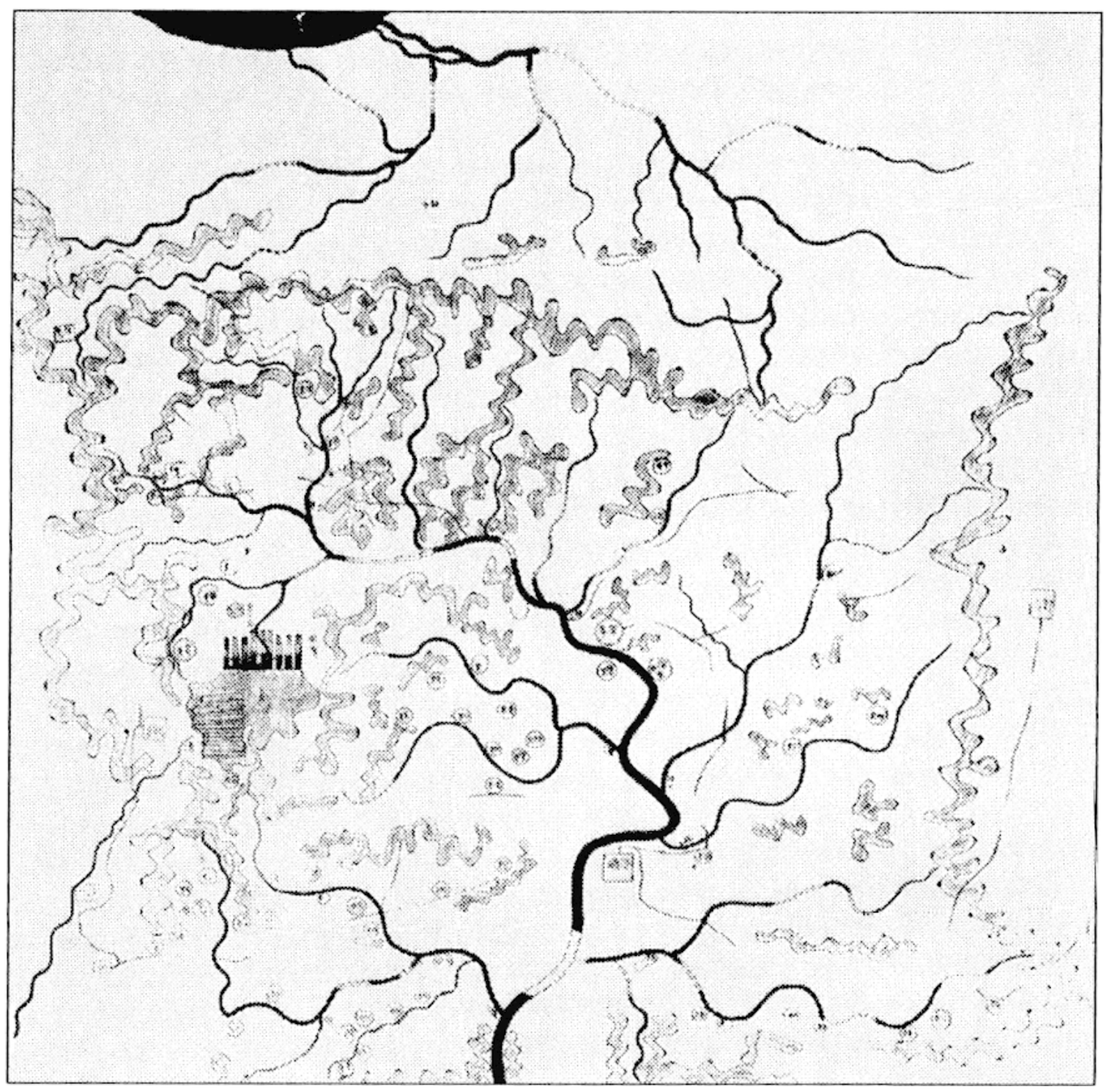

Carte des reliefs (Mawangdui, d'après Zhongguo gudai ditu ji, ill. 22). (D.R.) 
L'aménagement du territoire et la notion de frontière à l'époque ancienne

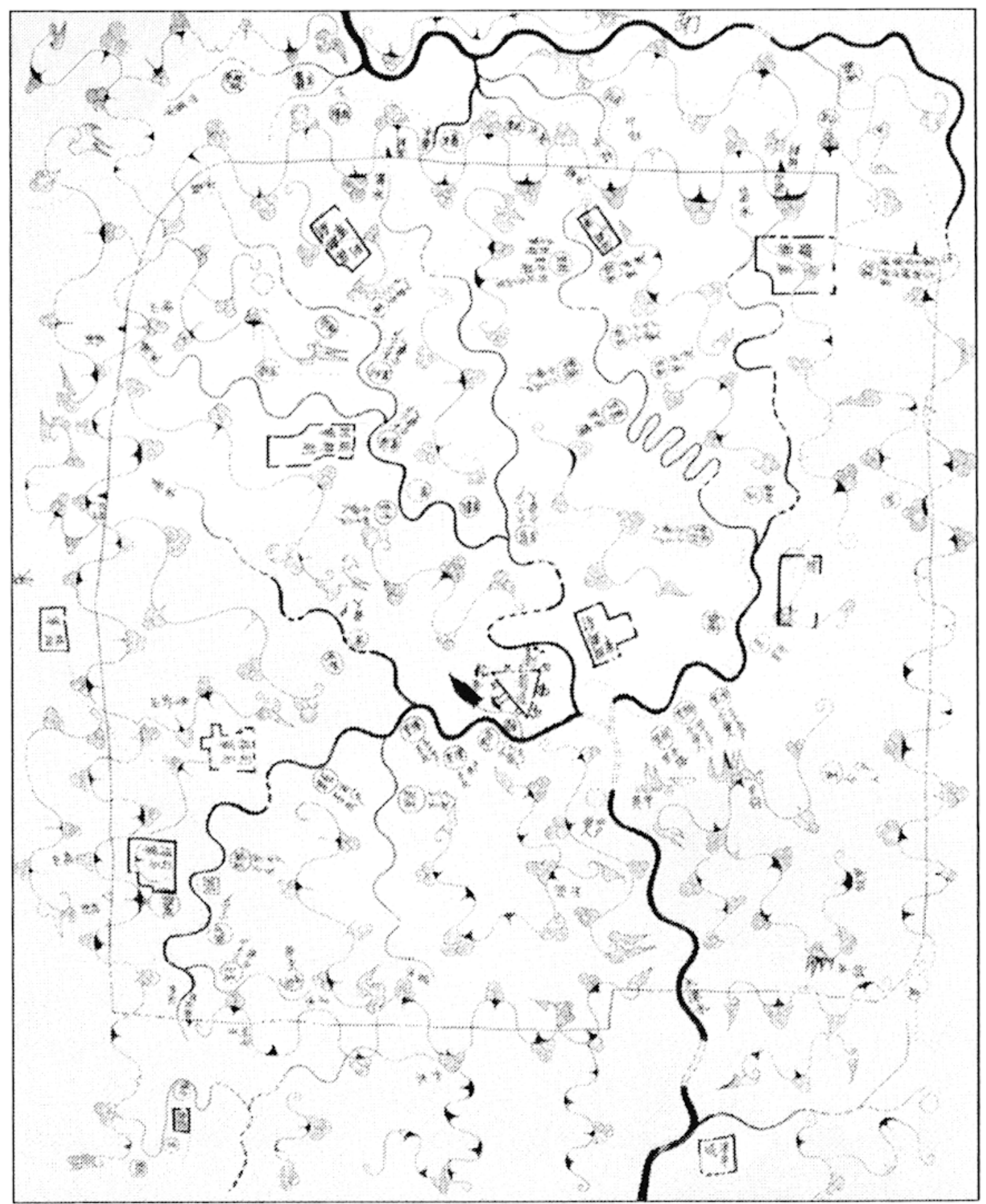

Carte des dispositifs de défense (Mawangdui, d'après Zhongguo gudai ditu ji, ill. 27). (D.R.) 